\title{
CHARACTERIZING THE SPECTRA OF CARDINALITIES OF BRANCHES OF KUREPA TREES
}

\author{
MÁRK POÓR* AND SAHARON SHELAH ${ }^{\dagger}$
}

\begin{abstract}
We give a complete characterization of the sets of cardinals that in a suitable forcing extension can be the Kurepa spectrum, that is, the set of cardinalities of branches of Kurepa trees. This answers a question of the first named author.
\end{abstract}

\section{INTRODUCTION}

A tree is a Kurepa tree if it is of height $\omega_{1}$, each of its levels is countable, and it has more than $\omega_{1}$-many cofinal (that is of order type $\omega_{1}$ ) branches. In this paper we study the possible values of the branch spectrum of Kurepa trees, i.e. the set

$$
\mathrm{Sp}_{\omega_{1}}=\{\lambda \text { : there exists a Kurepa tree } T \text { s.t. }|\mathcal{B}(T)|=\lambda\} \subseteq\left[\omega_{2}, 2^{\omega_{1}}\right]
$$

(where $\mathcal{B}(T)$ stands for the set of cofinal branches of $T$ ).

The spectrum is related to the model theoretical spectrum of maximal models of $\mathcal{L}_{\omega_{1}, \omega}$-sentences SS17. Also canonical topological and combinatorial structures are associated with branches of Kurepa trees possessing a remarkably wide range of nonreflecting properties Kos05. For higher Kurepa trees (of weakly compact height) the consistency strength of certain types of the branch spectrum was studied in HM19.

It was first shown by Silver that the Kurepa Hypothesis (i.e. the existence of a Kurepa tree) is independent [Sil67, or see [Kun83, Ch VIII, 3.]. Moreover the non-existence of Kurepa trees is equiconsistent with the existence of an inaccessible cardinal [Kun83, Ch VII, Ex. B8.].

Questions about the possible values of the spectrum were addressed by Jin and Shelah in [JS92. They proved (assuming an inaccessible cardinal) that consistently there are only Kurepa trees with $\omega_{3}$-many cofinal branches while $2^{\omega_{1}}=\omega_{4}$.

Building on ideas of Jin and Shelah, the first named author provided a sufficient condition for a set to be equal to $\mathrm{Sp}_{\omega_{1}}$ in a forcing extension in $\mathrm{PoO}$. Formally, it was shown that if $\mathbf{G C H}$ holds, and $0,1 \notin S$ is a set of ordinals such that $S$ satisfies either

Case A:

(i) $2 \in S$,

2010 Mathematics Subject Classification. Primary 03E35; Secondary 03E05, 03E45.

Key words and phrases. Kurepa tree, Constructible universe, Cardinal spectra.

* The first author was supported by the National Research, Development and Innovation Office - NKFIH, grants no. 124749, 129211.

${ }^{\dagger}$ The second author was partially supported by the European Research Council (ERC) grant 338821, and by the Israel Science Foundation grant 1838/19. Paper 1189 on Shelah's list. Supported by the ÚNKP-19-3 New National Excellence Program of the Ministry of Human Capacities. 
(ii) $\left\{\sup C: C \in[S] \leq \omega_{1}\right\} \subseteq S$,

(iii) $(\forall \alpha \in S):\left(\omega \leq \operatorname{cf}(\alpha)<\omega_{2}\right) \rightarrow(\alpha+1 \in S)$,

or

Case B:

(i) $\exists$ an inaccessible $\kappa$,

(ii) $\left\{\sup C: C \in[S]^{<\kappa}\right\} \subseteq S$,

(iii) $(\forall \alpha \in S):(\omega \leq \operatorname{cf}(\alpha)<\kappa) \rightarrow(\alpha+1 \in S)$,

then in a forcing extension we have $\left\{\alpha: \aleph_{\alpha} \in \operatorname{Sp}_{\omega_{1}}\right\}=S$ (cardinals are only collapsed in Case B, from $\left.\left(\omega_{1}, \kappa\right)\right)$. It can be easily seen that if $\operatorname{cf}(\mu)=\omega$ and $\left(\mathrm{Sp}_{\omega_{1}} \cap \mu\right)$ is cofinal in $\mu$, then there exists a Kurepa tree with $\mu$-many branches, as the union of countably many Kurepa trees is a Kurepa tree, and it is not difficult to see that the same holds if $\operatorname{cf}(\mu)=\omega_{1}$, therefore Case A / (ii) and Case B / (ii) are in fact necessary. However, it remained a question whether the last clauses can be dropped.

In this paper as the main result we prove that assuming $\mathbf{C H}+\left(2^{\omega_{1}}=\omega_{2}\right)$ conditions (i), (ii) (in both cases) are in fact sufficient by forcing a model of $\{\alpha$ : $\left.\aleph_{\alpha} \in \operatorname{Sp}_{\omega_{1}}\right\}=S$. Also, we can arbitrarily prescribe $2^{\omega_{1}}$ to be any cardinal $\lambda \geq$ $\sup \left(\operatorname{Sp}_{\omega_{1}}\right)$ if in Case A the equality $\lambda^{<\omega_{2}}=\lambda$ holds, or in Case $\mathrm{B} \lambda^{<\kappa}=\lambda$ holds too.

Moreover, when we do not want Kurepa trees with $\omega_{2}$-many cofinal branches, we prove that the inaccessible is necessary by verifying that if $\omega_{2}$ is a successor in $L$, then there exists a Kurepa tree with only $\omega_{2}$-many cofinal branches in $V$. It was known that these assumptions imply that there exists a Kurepa tree even in $L[A]$ for some $A \subseteq \omega_{1}$ Kun83, Ch VII, Ex. B8.] (possibly having more than $\omega_{2}$-many cofinal branches in $V$ ). Our proof not only utilizes countable elementary submodels of initial segments of $L[A]$, but the nodes of the tree are such elementary submodels, and each cofinal branch uniquely corresponds to an initial segment of $L[A]$.

\section{Preliminaries, notations}

Under ordinals we always mean Neumann ordinals. For a fixed cardinal $\chi$ we will use the notation $\mathcal{H}(\chi)$ for the collection of sets of hereditary size less than $\chi$, i.e.

$$
\mathcal{H}(\chi)=\{x:|\operatorname{trcl}(x)|<\chi\},
$$

where $\operatorname{trcl}(x)$ stands for the transitive closure of $x$. In terms of forcing we will use the notations of Kun13], e.g. $p \leq q$ means that $p$ is the stronger. If it is clear from the context and won't make any confusion we will identify the set $x$ in the ground model with its canonical name $\check{x}$. For a set $A$ the symbol $\mathcal{P}(A)$ denotes the powerset of $A$, and $[A]^{\lambda}$ stands for $\{X \in \mathcal{P}(A):|X|=\lambda\}$. For a function $s=\{\langle\beta, s(\beta)\rangle: \beta \in \operatorname{dom}(s)\}$ we will also use the following notation and refer to $s$ as

$$
\left\langle s_{\beta}: \beta \in \operatorname{dom}(s)\right\rangle .
$$

Under a sequence we mean a function defined on a set of ordinals. For sequences $s, t$ the relation $s=t\lceil\operatorname{dom}(s)$ (or equivalently $s \subseteq t$ ) will be also denoted by $s \triangleleft t$.

Definition 2.1. A tree $\left\langle T, \prec_{T}\right\rangle$ is a partially ordered set (poset) in which for each $x \in T$ the set

$$
T_{\prec x}=\left\{y \in T: y \prec_{T} x\right\}
$$



is well ordered by $\prec_{T}$.

Definition 2.2. The height of $x$ in the tree $T$ is the order type of $T_{\prec x}$

$$
\operatorname{ht}(x)=\operatorname{otp}\left(T_{\prec x}\right) .
$$

Definition 2.3. For each ordinal $\alpha$ the restriction of $T$ to $\alpha$ is

$$
T_{<\alpha}=\{t \in T: \operatorname{ht}(t)<\alpha\} .
$$

Definition 2.4. The height of the tree $T$ (in symbols $h t(T)$ ), is the least $\beta$ such that

$$
\nexists t \in T: \operatorname{ht}(t)=\beta .
$$

We will need the following lemma Kun83, Ch II. Thm. 1.6.] which we will refer to as the $\Delta$-system Lemma.

Lemma 2.5. Let $\kappa$ be an infinite cardinal, let $\theta>\kappa$ be regular, and satisfy $\forall \alpha<\theta$ $\left(\left|\alpha^{<\kappa}\right|<\theta\right)$. Assume that $|\mathcal{A}| \geq \theta$, and $\forall x \in \mathcal{A}(|x|<\kappa)$. Then there is a $\mathcal{D} \subseteq \mathcal{A}$, such that $|\mathcal{D}|=\theta$, and $\mathcal{D}$ forms a $\Delta$-system, i.e. there is a kernel set $y$ such that

$$
\forall x \neq x^{\prime} \in \mathcal{D}: \quad x \cap x^{\prime}=y .
$$

\section{THE FORCING}

Now we can state our main theorem.

Theorem 3.1. Let $S_{\bullet}$ be a set of infinite cardinals such that $\omega, \omega_{1} \notin S_{\bullet}$. Assume $\mathbf{C H}$, and that either

Case 1:

(i) $\omega_{2} \in S_{\bullet}$,

(ii) $2^{\omega_{1}}=\omega_{2}$,

(iii) $\left\{\sup C: C \in\left[S_{\bullet}\right]^{<\omega_{2}}\right\} \subseteq S_{\bullet}$,

or

Case 2:

(i) there exists an inaccessible $\kappa$ such that $S_{\bullet} \cap\left(\omega_{1}, \kappa\right)=\emptyset$,

(ii) $\left\{\sup C: C \in\left[S_{\bullet}\right]^{<\kappa}\right\} \subseteq S_{\bullet}$.

Then there exists a forcing extension $V^{\mathbb{P}}$ such that

$$
V^{\mathbb{P}} \models S_{\bullet}=\operatorname{Sp}_{\omega_{1}} \text {, where } \mathbb{P} \text { only collapses cardinals in }\left(\omega_{1}, \kappa\right) \text { in } \underline{\text { Case2 }} \text {. }
$$

The key will be Lemma 3.26. After Lemma 3.29 we will put together the pieces in a short argument. Before these we need some preparation.

Definition 3.2. In Case 1 (i.e. $\omega_{2} \in S_{\bullet}$ ) define the cardinal $\kappa$ to be $\omega_{2}$.

Corollary 3.3. No cardinal $\mu \notin\left(\omega_{1}, \kappa\right)$ is collapsed.

Theorem 3.4. Suppose that all conditions from Theorem 3.1 hold, and $\kappa$ is defined in Definition 3.2. Assume further that $\lambda$ is a cardinal which is an upper bound of $S$. such that $\lambda^{<\kappa}=\lambda$ (thus $\left.\operatorname{cf}(\lambda) \geq \kappa\right)$. Then there exists a forcing extension $V^{\mathbb{P}}$ with

$V^{\mathbb{P}} \models\left(S_{\bullet}=\{\mu\right.$ : there exists a Kurepa tree $T$ s.t. $\left.|\mathcal{B}(T)|=\mu\}\right) \wedge\left(2^{\omega_{1}}=\lambda\right)$.

Definition 3.5. Let $S_{\bullet}^{+}=S_{\bullet} \cup\{\kappa, \lambda\}$. 
Definition 3.6. For a cardinal $\theta \in S$. let $\mathbb{Q}_{\theta}$ be the following notion of forcing. The triplet $p=\left\langle T_{p}, u_{p}, \bar{\eta}_{p}\right\rangle$ is an element of $\mathbb{Q}_{\theta}$ iff

(a) $T_{p}$ is a countable tree of height $\delta$ for some $\delta<\omega_{1}$ on the underlying set $\omega \cdot \delta$, where the $\beta$ 'th level is $[\omega \cdot \beta, \omega \cdot(\beta+1))$, i.e. $T_{p, \leq \beta} \backslash T_{p,<\beta}=[\omega \cdot \beta, \omega \cdot(\beta+1))$ for each $\beta<\delta$,

(b) for each $t \in T_{p}$ and $\beta<\delta$ there exists $t^{\prime} \in T_{p} \backslash T_{p,<\beta}$ s.t. $t \prec_{T_{p}} t^{\prime}$,

(c) $u_{p} \in[\theta] \leq \omega$,

(d) $\bar{\eta}_{p}=\left\langle\eta_{p, \alpha}: \alpha \in u_{p}\right\rangle$, where $\eta_{p, \alpha} \subseteq T_{p}$ is a branch in $T_{p,<\gamma}$ for some $\gamma \in\left\{\beta+1: \beta<\delta=\operatorname{ht}\left(T_{p}\right)\right\}$ (we do it for a technical reason, we also could have stored only the maximal element instead of a chain with a maximal element).

Then $\mathbb{Q}_{\theta}$ is a poset with the obvious order, i.e. $q \leq p$, if $T_{q}$ is an end-extension of $T_{p}$, formally $T_{q,<\mathrm{ht}\left(T_{p}\right)}=T_{p}$, and for each $\alpha \in u_{p}$ the inclusion $\eta_{p, \alpha} \subseteq \eta_{q, \alpha}$ holds.

Let $\underset{\sim}{T}, \bar{\eta}_{\theta}$ be the names for the generic tree and sequence, i.e. denoting the generic filter by $\mathbf{G}_{\theta}$

$$
\begin{array}{ll}
1_{\mathbb{Q}_{\theta}} \Vdash & \underset{\sim}{T} \theta=\cup\left\{T_{p}: p \in \mathbf{G}_{\theta}\right\} \\
1_{\mathbb{Q}_{\theta}} \Vdash & \underset{\sim}{\sim}{ }_{\theta}=\left\langle\sim_{\theta, \alpha}=\cup\left\{\eta_{p, \alpha}: p \in \mathbf{G}_{\theta}\right\}: \alpha \in \theta\right\rangle .
\end{array}
$$

Definition 3.7. For a cardinal $\theta \in S_{\bullet}$ let $\mathbb{Q}_{\theta}^{*} \subseteq \mathbb{Q}_{\theta}$ be the following subposet.

$p \in \mathbb{Q}_{\theta}^{*}$, iff $\operatorname{ht}\left(T_{p}\right)$ is a successor, and $\left(\forall \alpha \in u_{p}\right): \eta_{p, \alpha}$ is a branch through $T_{p}$.

Definition 3.8. If $\lambda \notin S$. then let $\mathbb{Q}_{\lambda}$ be the countable supported product of $\left\langle<\omega_{1} 2, \triangleleft\right\rangle$-s of length $\lambda$, i.e.

$$
\mathbb{Q}_{\lambda}=\left\{p=\left\langle\eta_{\alpha}: \alpha \in u_{p}\right\rangle:\left(\forall \alpha \in u_{p}\right) \eta_{\alpha} \in<\omega_{1} 2, \text { for some } u_{p} \in[\lambda]^{\leq \omega}\right\} .
$$

Definition 3.9. If $\kappa \notin S$ • (and then $\kappa>\omega_{2}^{V}$ is inaccessible), then let $\mathbb{Q}_{\kappa}$ be the countable supported product of $\left\langle{ }^{<\omega_{1}} \gamma, \triangleleft\right\rangle$ 's $(\gamma<\kappa)$, a forcing which collapses each cardinal in $\left(\omega_{1}, \kappa\right)$ :

$$
\mathbb{Q}_{\kappa}=\left\{p=\left\langle\eta_{\alpha}: \alpha \in u_{p}\right\rangle:\left(\forall \alpha \in u_{p}\right) \eta_{\alpha} \in{ }^{<\omega_{1}} \alpha \text {, for some } u_{p} \in[\kappa]^{\leq \omega}\right\} .
$$

Definition 3.10. We define the posets which we will need later.

1) For $S \subseteq S_{\bullet}^{+}$let $\mathbb{P}_{S}$ be the countable supported product of $\mathbb{Q}_{\theta}$-S $(\theta \in S)$, i.e. $\mathbb{P}_{S}=\left\{p\right.$ is a function : $\left.\operatorname{dom}(p) \in[S] \leq \omega \wedge\left(\forall \theta \in \operatorname{dom}(p) p(\theta) \in \mathbb{Q}_{\theta}\right)\right\}$.

With a slight abuse of notation for $p \in \mathbb{P}_{S}$ and $\theta \in S \backslash \operatorname{dom}(p)$ we will mean $1_{\mathbb{Q}_{\theta}}$ under $p(\theta)$.

2) For $\theta \in S_{\bullet}^{+}, U \subseteq \theta$ define its restriction from $\theta$ to $U$, i.e.

$$
\mathbb{Q}_{\theta, U}=\left\{p \in \mathbb{Q}: u_{p} \subseteq U\right\} \text {. }
$$

3) For $S \subseteq S_{\bullet}^{+}, \bar{U}=\left\langle U_{\theta}: \theta \in S\right\rangle \in \prod_{\theta \in S} \mathcal{P}(\theta)$ we define $\mathbb{P}_{S, \bar{U}}$ to be $\mathbb{P}_{\text {- }}$ restriction to coordinates in $U_{\theta}$-s, i.e.

$$
\mathbb{P}_{S, \bar{U}}=\left\{p \in \mathbb{P}_{S}:(\forall \theta \in S) p(\theta) \in \mathbb{Q}_{\theta, U_{\theta}}\right\} .
$$

4) For $S, S^{\prime} \subseteq S_{\bullet}^{+}, \bar{U}=\left\langle U_{\theta}: \theta \in S\right\rangle \in \prod_{\theta \in S} \mathcal{P}(\theta), \bar{U}^{\prime}=\left\langle U_{\theta}^{\prime}: \theta \in S\right\rangle \in$ $\prod_{\theta \in S^{\prime}} \mathcal{P}(\theta)$ we define

- $\bar{U}+\bar{U}^{\prime}=\left\langle U_{\theta} \cup U_{\theta}^{\prime}: \theta \in S \cup S^{\prime}\right\rangle$ (where for $\theta \in S^{\prime} \backslash S$ under $U_{\theta}$ we mean the empty set, similarly for $\left.\theta \in S \backslash S^{\prime}, U_{\theta}^{\prime}\right)$, 
CHARACTERIZING THE SPECTRA OF CARDINALITIES OF BRANCHES OF KUREPA TREES

- $\bar{U}-\bar{U}^{\prime}=\left\langle U_{\theta} \backslash U_{\theta}^{\prime}: \theta \in S\right\rangle$ (here we also mean the empty set under $U_{\theta}^{\prime}$ if $\left.\theta \in S \backslash S^{\prime}\right)$,

- $\overline{\mathrm{id}}_{S}=\langle\theta: \theta \in S\rangle$

- for the set $X$ if $\bar{W}_{\alpha} \in \prod_{\theta \in S} \mathcal{P}(\theta)(\alpha \in X)$ then

$$
\sum_{\alpha \in X} \bar{W}_{\alpha}=\left\langle\bigcup_{\alpha \in X}\left(W_{\alpha}\right)_{\theta}: \theta \in S\right\rangle \text {. }
$$

5) Let $\mathbb{P}=\mathbb{P}_{S_{\bullet}^{+}}$.

6) If $p_{0}, p_{0}, \ldots, p_{n} \in \mathbb{P}$ let $\bigwedge_{i \leq n} p_{i}$ denote the greatest lower bound if exists.

7) For $p \in \mathbb{P}$, and $S \subseteq S_{\bullet}^{+}, \bar{U}=\left\langle U_{\theta}: \theta \in S\right\rangle \in \prod_{\theta \in S} \mathcal{P}(\theta)$ define $p\left\lceil\bar{U} \in \mathbb{P}_{S}\right.$ to be the following restriction of $p\lceil S$ in the obvious fashion

$$
\text { for each } \theta \in S: \quad\left(p\lceil\bar{U})(\theta)=\left\langle T_{p(\theta)}, u_{p_{\theta}} \cap U_{\theta}, \bar{\eta}_{p}\left\lceil U_{\theta}\right\rangle\right. \text {. }\right.
$$

Definition 3.11. For $S \subseteq S_{\bullet}^{+}$define the notion of forcing $\mathbb{P}^{*}\left(\mathbb{P}_{S}^{*}, \mathbb{P}_{S, \bar{U}}^{*}\right.$, resp. $)$ to be the subposet of $\mathbb{P}\left(\mathbb{P}_{S}, \mathbb{P}_{S, \bar{U}}\right.$, resp.) consisting of elements $p$ for that $p(\theta) \in \mathbb{Q}_{\theta}^{*}$ holds for each $\theta \in S_{\bullet} \cap \operatorname{supp}(p)$.

Remark 3.12. The notion of forcing $\mathbb{P}^{*}\left(\mathbb{P}_{S}^{*}, \mathbb{P}_{S, \bar{U}}^{*}\right.$, resp.) is a dense subposet of $\mathbb{P}\left(\mathbb{P}_{S}, \mathbb{P}_{S, \bar{U}}\right.$, resp.), therefore forcing with $\mathbb{P}^{*}\left(\mathbb{P}_{S}^{*}, \mathbb{P}_{S, \bar{U}}^{*}\right.$, resp. $)$ yields the same extensions as forcing with $\mathbb{P}\left(\mathbb{P}_{S}, \mathbb{P}_{S, \bar{U}}\right.$, resp.).

Claim 3.13. Let $S \subseteq S_{\bullet}^{+}, \bar{U}=\left\langle U_{\theta}: \theta \in S\right\rangle$ be fixed. Then the poset $\mathbb{P}_{S, \bar{U}}$ has the $\kappa$-cc property.

Proof. Suppose that $\left\{p_{\alpha}: \alpha \in \kappa\right\} \subseteq \mathbb{P}_{S, \bar{U}}$ is an antichain. Working in $V^{\prime}$, applying the $\Delta$-system lemma (Lemma 2.5) for the system $\left\{\operatorname{dom}\left(p_{\alpha}\right): \alpha \in \kappa\right\}$ of countable sets (1) from Definition 3.10), we obtain a set $A \in[\kappa]^{\kappa}$, such that the $\operatorname{dom}\left(p_{\alpha}\right)$ 's $(\alpha \in A)$ form a $\Delta$-system with kernel $K \subseteq S$. Since $K$ is obviously countable, for each $\alpha$ we have that $\left\langle T_{p_{\alpha}(\theta)}: \theta \in K\right\rangle$ is a countable sequence of countable trees (by (a) from Definition 3.6). This means that by $\mathbf{C H}$ we can assume that

$$
\left\langle T_{p_{\alpha}(\theta)}: \theta \in K\right\rangle=\left\langle T_{p_{\beta}(\theta)}: \theta \in K\right\rangle \quad(\forall \alpha, \beta \in A) .
$$

Now applying the $\Delta$-system lemma again for the system

$$
U_{\alpha}=\bigcup_{\theta \in S}\left(\{\theta\} \times u_{p_{\alpha}(\theta)}\right) \quad(\alpha \in \kappa)
$$

yields a set $A^{\prime} \in[A]^{\kappa}$ such that the $U_{\alpha}$ 's $\left(\alpha \in A^{\prime}\right)$ form a $\Delta$-system with kernel $I \subseteq \bigcup_{\theta \in S}\{\theta\} \times \theta$ (of course, in fact, $I \subseteq \bigcup_{\theta \in K}\{\theta\} \times \theta$ ). Now by (3.1) it suffices to prove that

$$
\exists \alpha \neq \beta \in A^{\prime} \text { such that (for each }\langle\theta, \delta\rangle \in I \text { ) : } \quad \eta_{p_{\alpha}(\theta), \gamma}=\eta_{p_{\beta}(\theta), \gamma},
$$

for which it is enough to prove

$$
\left|\left\{\left\langle\eta_{p_{\alpha}(\theta), \gamma}:\langle\theta, \gamma\rangle \in I\right\rangle: \alpha \in A^{\prime}\right\}\right|<\kappa .
$$

Fix $\alpha \in A^{\prime}$. Now for each $\langle\theta, \gamma\rangle \in I$, if $\theta \in S_{\bullet}$ then $\eta_{p_{\alpha}(\theta), \gamma} \in\left[\omega_{1}\right]^{<\omega_{1}}$ (a branch through $\left.T_{p_{\alpha}(\theta)}\right)$.

This means that (using that $I$ is countable)

$$
\left\{\left\langle\eta_{p_{\alpha}(\theta), \gamma}:\langle\theta, \gamma\rangle \in I, \theta \in S_{\bullet}\right\rangle: \alpha \in A^{\prime}\right\} \subseteq \prod_{\langle\theta, \gamma\rangle \in I, \theta \in S_{\bullet}}\left[\omega_{1}\right]^{<\omega_{1}}
$$


which latter set is of size $\omega_{1}$ by $\mathbf{C H}$. Second, if $\theta=\lambda \in\left(S_{\bullet}^{+} \backslash S_{\bullet}\right) \cap S$, then

$$
\left\{\left\langle\eta_{p_{\alpha}(\theta), \gamma}:\langle\theta, \gamma\rangle \in I, \theta=\lambda\right\rangle: \alpha \in A^{\prime}\right\} \subseteq \prod_{\langle\theta, \gamma\rangle \in I, \theta=\lambda}<\omega_{1} 2 .
$$

Finally we have to consider the coordinate $\theta=\kappa$ if $\kappa \in S \backslash S_{\bullet}$. Then letting $\delta=\sup \{\gamma:\langle\kappa, \gamma\rangle \in I\}$ we have $\delta<\kappa$, because $I$ is countable and $\kappa$ is inaccessible. Then

$$
\left\{\left\langle\eta_{p_{\alpha}(\kappa), \gamma}:\langle\kappa, \gamma\rangle \in I\right\} \subseteq \prod_{\langle\kappa, \gamma\rangle \in I}{ }^{<\omega_{1}} \delta\right.
$$

and since $\kappa$ is inaccessible, this case $\left|\prod_{\langle\kappa, \gamma\rangle \in I}<\omega_{1} \delta\right|<\kappa$. We obtain (using $\omega_{1}<\kappa$ ) that

$$
\mid\left\{\left\langle\eta_{p_{\alpha}(\theta), \gamma}:\langle\theta, \gamma\rangle \in I\right\}\left|\leq \omega_{1} \cdot \omega_{1} \cdot\right| \prod_{\langle\kappa, \gamma\rangle \in I}<\omega_{1} \delta \mid<\kappa,\right.
$$

therefore (3.3) holds.

Now we make the intuition behind the easy idea of first adding the trees and some branches, and then forcing over the extension precise.

Claim 3.14. For each $S \subseteq S_{\bullet}^{+}, \bar{U}=\left\langle U_{\theta}: \theta \in S\right\rangle$ we have

$$
\mathbb{P}_{S, \bar{U}} \lessdot \mathbb{P}_{S} \lessdot \mathbb{P},
$$

i.e. $\mathbb{P}_{S, \bar{U}}$ completely embeds into $\mathbb{P}_{S}$, which completely embeds into $\mathbb{P}$.

Proof. Since $\mathbb{P} \simeq \mathbb{P}_{S} \times \mathbb{P}_{S_{\bullet}^{+} \backslash S}$, it is enough to prove that $\mathbb{P}_{S, \bar{U}} \lessdot \mathbb{P}_{S}$.

Assume that $A \subseteq \mathbb{P}_{S, \bar{U}}$ is a maximal antichain in $\mathbb{P}_{S, \bar{U}}$, and let $p \in \mathbb{P}_{S} \backslash \mathbb{P}_{S, \bar{U}}$. Then there exists $a \in A, a^{\prime} \in \mathbb{P}_{S, \bar{U}}$ such that $a^{\prime} \leq a, a^{\prime} \leq b \uparrow \bar{U}$. But then it is straightforward to check that also $a^{\prime}$ and $b$ have a common lower bound.

Definition 3.15. Let $S \subseteq S_{\bullet}, \bar{U}=\left\langle U_{\theta}: \theta \in S\right\rangle, \theta_{0} \in S, U_{\theta_{0}}^{\prime} \subseteq \theta_{0} \backslash U_{\theta_{0}}$. Then

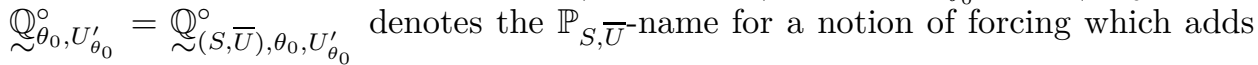
the branches $\underset{\sim}{\sim} \theta_{0}, \alpha\left(\alpha \in U_{\theta_{0}}^{\prime}\right)$ to $\underset{T_{\theta_{0}}}{\sim}$ in the following way

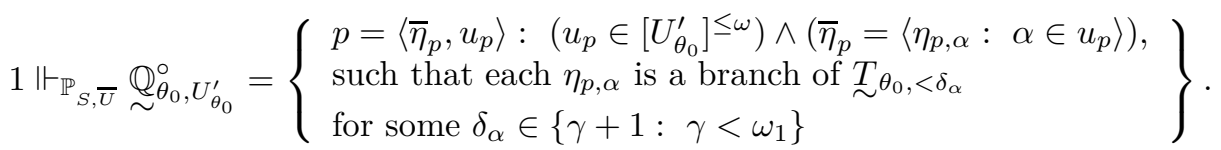

If it is clear from the context we will use $\mathbb{Q}_{\theta_{0}, U_{\theta_{0}}^{\prime}}^{\circ}$ not mentioning $S$ and $\bar{U}$.

Definition 3.16. Let $S \subseteq S_{\bullet}, \bar{U}=\left\langle U_{\theta}: \theta \in S\right\rangle, \theta_{0} \in S$.

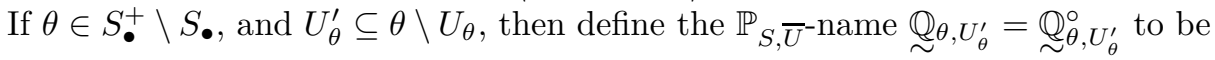
the name for $\mathbb{Q}_{\theta, U_{\theta}^{\prime}}$.

Definition 3.17. Let $S \subseteq S_{\bullet}^{+}, \bar{U}=\left\langle U_{\theta}: \theta \in S\right\rangle, \bar{U}^{\prime}=\left\langle U_{\theta}^{\prime}: \theta \in S\right\rangle \in \prod_{\theta \in S} \mathcal{P}(\theta)$, where $U_{\theta} \cap U_{\theta}^{\prime}=\emptyset$ for each $\theta \in S$. Then $\underset{\sim}{\mathbb{P}}{\overline{U^{\prime}}}^{\prime}=\underset{\sim}{\mathbb{P}}(S, \bar{U}), \bar{U}^{\prime}$ denotes the $\mathbb{P}_{S, \bar{U}^{-} \text {-name for }}$ the countably supported product of $\mathbb{Q}_{\theta, U_{\theta}^{\prime}}^{\circ}$ 's $(\theta \in S)$, i.e. a notion of forcing which 
adds the branches $\underset{\sim}{\eta_{\theta, \alpha}}\left(\alpha \in U_{\theta}^{\prime}\right)$ to $\underset{\sim}{\underset{\sim}{\sim} \theta}$ for each $\theta \in S \backslash S_{\bullet}$, and the sequences $\stackrel{\eta}{\sim}_{\kappa, \alpha}$ $\left(\alpha \in U_{\kappa}^{\prime}\right)$ if $\kappa \in S \backslash S_{\bullet}, \eta_{\lambda, \alpha}\left(\alpha \in U_{\lambda}^{\prime}\right)$ if $\lambda \in S \backslash S_{\bullet}:$

$1 \Vdash_{\mathbb{P}_{S, \bar{U}}} \stackrel{\mathbb{P}}{\sim}{\overline{U^{\prime}}}^{\circ}=\left\{p\right.$ is a function : $\left.\left.\operatorname{dom}(p) \in[S] \leq \omega \wedge\left(\forall \theta \in \operatorname{dom}(p) p(\theta) \in \mathbb{Q}_{\theta}^{\circ}, U_{\theta}^{\prime}\right)\right\}\right\}$.

Again, as in Definition 3.15 if it does not cause any confusion we only use the notation $\underset{\sim}{\stackrel{\circ}{\sim}} \bar{U}^{\prime}$ not mentioning $S$ and $\bar{U}$.

The following claim is an easy observation.

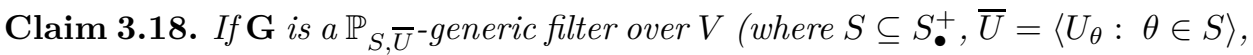
$\bar{U}^{\prime}=\left\langle U_{\theta}^{\prime}: \theta \in S\right\rangle \in \prod_{\theta \in S} \mathcal{P}(\theta)$, and $U_{\theta} \cap U_{\theta}^{\prime}=\emptyset$ for each $\left.\theta \in S\right)$, then with the notation from Kun13.

$$
\mathbb{P}_{S, \bar{U}+\bar{U}^{\prime}} / \mathbf{G}=\left\{p \in \mathbb{P}_{S, \bar{U}+\bar{U}^{\prime}}: \forall q \in \mathbf{G} p \not \perp q\right\},
$$

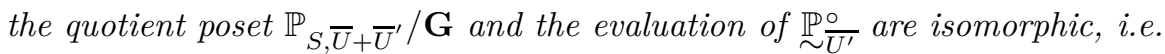

$$
V[\mathbf{G}] \models \underset{\sim}{\mathbb{P}} \stackrel{\circ}{\sim}[\mathbf{G}] \simeq \mathbb{P}_{S, \bar{U}+\bar{U}^{\prime}} / \mathbf{G} .
$$

Since $\mathbb{P}_{S, \bar{U}}$ completely embeds into $\mathbb{P}_{S, \bar{U}+\bar{U}^{\prime}}$ (by Claim 3.14), Kun13] [Lemma V.4.45.] (and [Kun13, Lemma V.4.44.]) implies the following.

Claim 3.19. Let $S \subseteq S_{\bullet}^{+}, \bar{U}=\left\langle U_{\theta}: \theta \in S\right\rangle, \bar{U}^{\prime}=\left\langle U_{\theta}^{\prime}: \quad \theta \in S\right\rangle \in \prod_{\theta \in S} \mathcal{P}(\theta)$, where $U_{\theta} \cap U_{\theta}^{\prime}=\emptyset$ for each $\theta \in S$. Then the canonical embedding from $\mathbb{P}_{S, \bar{U}+\bar{U}^{\prime}}$ to the iteration $\mathbb{P}_{S, \bar{U}} *\left(\mathbb{P}_{S, \bar{U}+\bar{U}^{\prime}} / \mathbf{G}\right)$ is a dense embedding.

Now putting together Claims 3.18 and 3.19 we have the following, meaning that instead of forcing with $\mathbb{P}_{S, \bar{U}+\bar{U}^{\prime}}$ we can force with $\mathbb{P}_{S, \bar{U}}$ and then with (the evaluation of) $\underset{\mathbb{P}}{\sim} \bar{U}^{\prime}$

Lemma 3.20. Let $S \subseteq S_{\bullet}^{+}, \bar{U}=\left\langle U_{\theta}: \theta \in S\right\rangle, \bar{U}^{\prime}=\left\langle U_{\theta}^{\prime}: \theta \in S\right\rangle \in \prod_{\theta \in S} \mathcal{P}(\theta)$, where $U_{\theta} \cap U_{\theta}^{\prime}=\emptyset$ for each $\theta \in S$. Then forcing with $\mathbb{P}_{S, \bar{U}+\bar{U}^{\prime}}$ amounts to the same as forcing with $\mathbb{P}_{S, \bar{U}}$ and then with $\mathbb{P}_{S, \bar{U}+\bar{U}^{\prime}} / \mathbf{G} \simeq \underset{\sim}{\mathbb{P}} \bar{U}^{\prime}$.

Definition 3.21. If $S \subseteq S_{\bullet}^{+}, \bar{U}=\left\langle U_{\theta}: \theta \in S\right\rangle, \bar{U}^{\prime}=\left\langle U_{\theta}^{\prime}: \theta \in S\right\rangle \in \prod_{\theta \in S} \mathcal{P}(\theta)$. Now if $\mathbf{G}$ is generic over $\mathbb{P}=\mathbb{P}_{S_{\bullet}^{+}}$then we define

- $\mathbf{G}_{S}=\mathbf{G} \cap \mathbb{P}_{S}$,

- $\mathbf{G}_{S, \bar{U}}=\mathbf{G} \cap \mathbb{P}_{S, \bar{U}}$,

- and $\mathbf{G}_{\bar{U}^{\prime}}^{\circ} \subseteq \mathbb{P}_{\bar{U}^{\prime}}^{\circ}\left[G_{S, \bar{U}}\right] \in V\left[G_{S, \bar{U}}\right]$ to be the filter given by the canonical mapping from Claims 3.18 , 3.19,

The following are basic observations. Roughly speaking, we isolate a dense $\omega_{1}$ closed subset of a two-step iteration similarly as in [Kun78].

Claim 3.22. $\mathbb{P}^{*}$ (and in general each $\mathbb{P}_{S, \bar{U}}^{*}$ ) is $\omega_{1}$-closed, i.e. for each decreasing sequence of type $\omega$ has a lower bound. In particular if $\mathbf{G}^{*} \subseteq \mathbb{P}^{*}$, (or in general $\left.\mathbf{G}_{S, \bar{U}}^{*} \subseteq \mathbb{P}_{S, \bar{U}}^{*}\right)$ is generic over $V$, then there is no new sequence of ordinals of type $\omega$.

The last claim and Remark 3.12 obviously implies the following. 
Claim 3.23. Forcing with $\mathbb{P}$ (or $\mathbb{P}_{S, \bar{U}}$ ) doesn't add new sequence of ordinals of type $\omega$, and for a given generic filter $\mathbf{G} \subseteq \mathbb{P}$

$$
\mathcal{H}\left(\omega_{1}\right)^{V}=\mathcal{H}\left(\omega_{1}\right)^{V[\mathbf{G}]}=\mathcal{H}^{V\left[\mathbf{G}_{S, \bar{U}}\right]} .
$$

Lemma 3.24. Let $\mathbf{G} \subseteq \mathbb{P}_{S, \bar{U}}$ generic over $V, B \in V[\mathbf{G}]$ where $B \subseteq \mathcal{H}\left(\omega_{1}\right)$. Then (in $V$ ) there exist $S_{*} \subseteq S,\left|S_{*}\right|<\kappa$ and $\bar{W}_{*}=\left\langle W_{\gamma}^{*}: \gamma \in S_{*}\right\rangle \in \prod_{\gamma \in S_{*}}\left[U_{\gamma}\right]^{<\kappa}$, such that $B \in V\left[\mathbf{G}_{S_{*}, \bar{W}_{*}}\right]$.

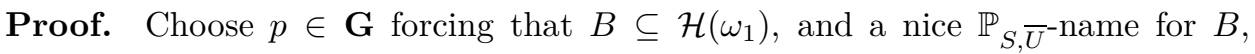
obtaining for each $x \in \mathcal{H}\left(\omega_{1}\right)$ an antichain $A_{x} \subseteq \mathbb{P}_{S, \bar{U}}$ deciding about $x \in B$. Then by $\kappa$-cc we have that each $\left|A_{x}\right|<\kappa$, the set $S_{*}=\bigcup_{x \in \mathcal{H}\left(\omega_{1}\right)} \bigcup_{a \in A_{x}} \operatorname{dom}(a)$ is of size less than $\kappa$ (as $\kappa$ is either inaccessible, or $\omega_{2}$ ). Also for $\theta \in S_{*}$ the set $W_{\theta}^{*}=\bigcup_{x \in \mathcal{H}\left(\omega_{1}\right)} \bigcup_{a \in A_{x}} u_{a(\theta)}$ is smaller that $\kappa$. Now it is easy to see that $\bar{W}_{*}=\left\langle W_{\gamma}^{*}: \gamma \in S_{*}\right\rangle$ is as claimed.

Then the following immediately follows from the $\omega_{1}$-closedness, and $\kappa$-cc.

Claim 3.25. Forcing with $\mathbb{P}$ doesn't collapse $\omega_{1}$, and cardinals at least $\kappa$. Moreover, if $\mathbf{G} \subseteq \mathbb{P}$ is generic, then

$$
V[\mathbf{G}] \models " \kappa=\omega_{2} " .
$$

Lemma 3.26. Let $T \in V\left[\mathbf{G}_{S, \bar{U}_{*}}\right]$ be a Kurepa tree, $S^{\prime} \subseteq S\left(S^{\prime} \in V\right)$. Then, if $b \in V\left[\mathbf{G}_{S, \bar{U}_{*}+\mathrm{id}_{S^{\prime}}}\right]$ is a branch of $T$, then there exists a finite set $S^{\prime \prime} \subseteq S^{\prime}$, and $\bar{U}_{\bullet}=\left\langle U_{\theta}^{\bullet}: \theta \in S^{\prime \prime}\right\rangle$ s.t. each $U_{\theta}^{\bullet}$ is finite, and $b$ is in the model obtained by adding these finitely many $\eta_{\theta, \alpha}$ 's $\left(\theta \in S^{\prime \prime}, \alpha \in U_{\theta}^{\bullet}\right)$ to $V\left[\mathbf{G}_{S, \bar{U}_{*}}\right]$, i.e.

\section{Proof.}

$$
b \in V\left[\mathbf{G}_{S, \bar{U}_{*}+\bar{U}_{\bullet}}\right] .
$$

Let $\dot{T} \in V$ be a $\mathbb{P}_{S, \bar{U}_{*}}$-name for $T$. Define

$$
\mathbb{P}^{\prime}=\mathbb{P}_{S, \bar{U}_{*}+\overline{\mathrm{id}}_{S^{\prime}}} .
$$

Suppose that $p_{*} \in \mathbb{P}^{\prime}$ forces that $\dot{b} \in V$ is a $\mathbb{P}^{\prime}$-name for a counterexample (i.e.

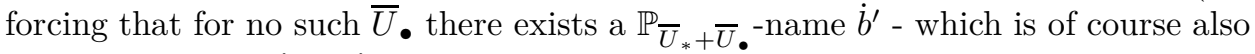
a $\mathbb{P}^{\prime}$-name - with $\dot{b}^{\prime}=\dot{b}$ ). Let $\chi$ be large enough, and let $\left\langle N_{0}, \in\right\rangle \prec\langle\mathcal{H}(\chi), \in\rangle$ be countable s.t. $p_{*}, \dot{b}, \dot{T}, S, S^{\prime}, \bar{V}, \mathbb{P}_{S, \bar{U}_{*}} \in N_{0}$.

Let $\delta_{\bullet}=N_{0} \cap \omega_{1}$. Define the countable set $N_{1}$ to be such that $N_{0} \in N_{1}$, and $\left\langle N_{1}, \in\right\rangle \prec\langle\mathcal{H}(\chi), \in\rangle$. Let $X$ be set of the indices of the new branches added to $\left\langle{\underset{\sim}{T} \theta}_{\theta}: \theta \in S^{\prime}\right\rangle$ by $\mathbf{G}_{S, \bar{U}_{*}+\left(\mathrm{id}_{S^{\prime}}\right)}$ that are in $V\left[\mathbf{G}_{S, \bar{U}_{*}+\overline{\mathrm{id}}_{S^{\prime}}}\right] \backslash V\left[\mathbf{G}_{S, \bar{U}_{*}}\right]$, and belong to $N_{0}$, i.e.

$$
X=N_{0} \cap\left\{\langle\theta, \alpha\rangle:\left(\theta \in S^{\prime}\right) \wedge\left(\alpha \in \theta \backslash U_{\theta}^{*}\right)\right\} .
$$

We fix an enumeration of $X$ and define also the sequence of the first $n$ indices from this countable set, and as well for each $n$ the one-length sequence consisting only the $n$ 'th, that is

$$
\begin{aligned}
& \text { let }\left\langle\left\langle\varrho_{n}, \xi_{n}\right\rangle: n \in \omega, n>0\right\rangle \text { enumerate } X \text { (starting from 1), } \\
& \bar{W}_{n}=\left\langle W_{n, \theta}: \theta \in S^{\prime} \cap N_{0}\right\rangle, \\
& \text { where } W_{n, \theta}=\left\{\alpha:\langle\theta, \alpha\rangle=\left\langle\varrho_{j}, \xi_{j}\right\rangle \text { for some } j \leq n\right\} \\
& \bar{w}_{n}=\left\langle w_{n, \theta}: \quad \theta \in S^{\prime} \cap N_{0}\right\rangle \\
& \text { where } w_{n, \theta}=\left\{\xi_{n}\right\} \text { if } \theta=\varrho_{n}, w_{n, \theta}=\emptyset \text { otherwise. }
\end{aligned}
$$


CHARACTERIZING THE SPECTRA OF CARDINALITIES OF BRANCHES OF KUREPA TREE9

Observe that if $p \in \mathbb{P} \cap N_{0}$, then each $\theta \in \operatorname{dom}(p)$ is an element of $N_{0}$ since $\operatorname{dom}(p)$ is countable (by Definition 3.10), and similarly $T_{p(\theta)}, u_{p(\theta)} \subseteq N_{0}$ (by Definitions $3.6-3.9$ ).

Working in $V$ we will construct an $N_{0}$-generic condition in $\mathbb{P}^{\prime}$, which will derive us to a contradiction. It is enough to prove the following claim.

Claim 3.27. There exists a sequence $\left\langle\bar{p}_{n}: n \in \omega\right\rangle \in V, p_{0}^{\prime} \in \mathbb{P}_{S, \bar{U} *}$ and a sequence $\bar{q}=\left\langle q_{n}: n \in \omega\right\rangle$ such that the following holds.

$\boxplus_{1} \bar{p}_{0}=\left\langle p_{0, l}: l \in \omega\right\rangle$ is such that

(a) $p_{0,0}=p_{*} \uparrow \bar{U}_{*}$,

(b) $p_{0, l} \in N_{0} \cap \mathbb{P}_{S, \bar{U}_{*}}$ for each $l \in \omega$,

(c) $\left\langle p_{0, l}: l \in \omega\right\rangle$ is $\leq_{\mathbb{P}}$-decreasing,

(d) $\bar{p}_{0} \in N_{1}$,

(e) letting $\mathbf{G}_{0}=\left\{p \in \mathbb{P}_{S, \bar{U} *} \cap N_{0}:(\exists l) p \geq p_{0, l}\right\}$, the filter $\mathbf{G}_{0}$ is $\mathbb{P}_{S, \bar{U}_{*}}$ generic over $N_{0}$.

$\boxplus_{2} p_{0}^{\prime} \in \mathbb{P}_{S, \bar{U} *}$ satisfies the following

(a) $p_{0}^{\prime}$ is a lower bound of $p_{0, l}$ for each $l \in \omega$ (hence forces a value to $\underset{\sim}{T} \theta,<\delta$. for each $\theta \in S \cap N_{0}$ ),

(b) $p_{0}^{\prime}$ forces a value to $\underset{\sim}{T} \theta, \leq \delta$. for each $\theta \in S \cap N_{0}$ such that for every $\delta_{\bullet}$-branch $B$ in $\underset{\sim}{T} \theta,<\delta_{\bullet}$ the inclusion $B \in N_{1}$ implies that $B$ has an upper bound in $\underset{\sim}{T} \theta, \leq \delta_{\bullet}$,

(c) $p_{0}^{\prime}$ forces a value to $\dot{T}_{\leq \delta_{\bullet}}$.

$\boxplus_{3}$ for every $n>0$ the sequence $\bar{p}_{n}=\left\langle p_{n, l}: l \in \omega\right\rangle$ has the following properties.

(a) $\forall l \in \omega p_{n, l} \in N_{0} \cap \mathbb{P}_{S, \bar{U}_{*}+\bar{w}_{n}}$,

(b) $p_{n, l}\left\lceil\bar{U}_{*} \in \mathbf{G}_{0}\right.$

(c) $\left\langle p_{n, l}: l \in \omega\right\rangle$ is $\leq_{\mathbb{P}}$-decreasing,

(d) $\bar{p}_{n} \in N_{1}$,

(e) letting

$$
\mathbf{G}_{n}=\left\{p \in \mathbb{P}_{S, \bar{U} *+\bar{W}_{n}} \cap N_{0}:\left(\exists l_{0}, l_{1}, \ldots, l_{n}\right) p \geq \bigwedge_{j=0}^{n} p_{j, l_{j}}\right\},
$$

the filter $\mathbf{G}_{n}$ is $\mathbb{P}_{S, \bar{U}_{*}+\bar{W}_{n}}$-generic over $N_{0}$.

$\boxplus_{4}$ For the sequence $\bar{q}=\left\langle q_{n}: n \in \omega\right\rangle$

(a) $q_{n} \in N_{0} \cap \mathbb{P}_{S, \bar{U}_{*}+\overline{\mathrm{id}}_{S^{\prime}}}$ for each $n \in \omega$,

(b) $q_{0}=p_{*}$,

(c) $\left\langle q_{n}: n \in \omega\right\rangle$ is $\leq_{\mathbb{P}}$-decreasing,

(d) $\forall n: q_{n} \uparrow\left(\bar{U}_{*}+\bar{W}_{n}\right) \in \mathbf{G}_{n}$,

(e) Let $\left\langle\dot{B}_{n}: n \in \omega\right\rangle$ enumerate the branches of $\dot{T}_{<\delta}$. which has an upper bound in $\dot{T}_{\leq \delta}$ (forced by $p_{0}^{\prime}$ ). Then $q_{n+1} \wedge p_{0}^{\prime}$ forces that $\dot{b} \neq B_{n}$, which will be guaranteed by the following requirement:

There exist $\delta<\delta_{\bullet}, t \neq t^{\prime} \in \dot{T}_{\leq \delta} \backslash \dot{T}_{<\delta}$, such that $p_{0}^{\prime}$ forces $B_{n}$-s $\delta$ 'th level to be $t^{\prime}$, and $q_{n+1}$ forces $t \in \dot{b}$, i.e.

$$
\begin{aligned}
& p_{0}^{\prime} \Vdash \dot{B}_{n} \cap\left(\dot{T}_{\leq \delta} \backslash \dot{T}_{<\delta}\right)=\left\{t^{\prime}\right\} \\
& \text { and } \\
& q_{n+1} \Vdash \dot{b} \cap\left(\dot{T}_{\leq \delta} \backslash \dot{T}_{<\delta}\right)=\{t\} .
\end{aligned}
$$

(Observe that the latter is a statement in $N_{0}$.) 
Before proving Claim 3.27 we argue why this claim implies Lemma 3.26. First,

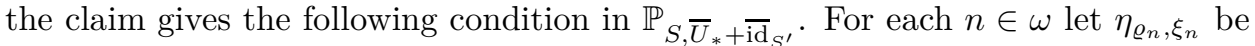
the branch in $\underset{\sim}{T} \varrho_{n},<\delta$. represented by the sequence $\bar{p}_{n}$, i.e.

$$
\eta_{\varrho_{n}, \xi_{n}}=\cup\left\{\eta_{p_{n, l}\left(\varrho_{n}\right), \xi_{n}}: l \in \omega\right\},
$$

and note that $\eta_{\varrho_{n}, \xi_{n}} \in N_{1}(n \in \omega)$ by $\mathbb{⿴ 囗 十 3}_{3} \sqrt{(d)}$. Therefore by $\mathbb{A}_{2} \sqrt{(b)}$ we can extend

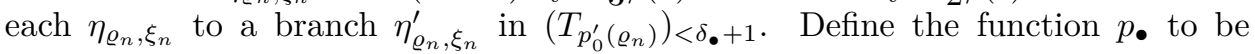
the extension of $p_{0}^{\prime}$ by the $\eta_{\varrho_{n}, \xi_{n}}$ 's in the obvious way: (Note that by $⿴ 囗 十 2$ we have $S \cap N_{0} \subseteq \operatorname{dom}\left(p_{0}^{\prime}\right) \subseteq S$, and for each $\theta \in S \cap N_{0}$ the inclusion $\left.U_{\theta}^{*} \cap N_{0} \subseteq u_{p_{0}^{\prime}(\theta)} \subseteq U_{\theta}^{*}.\right)$ Define $p_{\bullet}$ to be function on $\operatorname{dom}\left(p_{0}^{\prime}\right)$ such that if $\theta \notin N_{0} \cap S^{\prime}$, then $p_{\bullet}(\theta)=p_{0}^{\prime}(\theta)$, and for $\theta \in N_{0} \cap S^{\prime}$ define $p_{\bullet}(\theta)$ to be the following proper extension of $p_{0}^{\prime}(\theta)$. Let $u_{p_{\bullet}(\theta)}=u_{p_{0}(\theta)} \cup\left(\theta \cap N_{0}\right)$, and if $\alpha \notin u_{p_{0}^{\prime}(\theta)}$ (when necessarily $\alpha \notin U_{\theta}^{*}$ ) and (by (3.8) ) choose $n>0$ so that

$$
\langle\theta, \alpha\rangle=\left\langle\varrho_{n}, \xi_{n}\right\rangle, \text { and let } \eta_{p_{\bullet}(\theta), \alpha}=\eta_{\varrho_{n}, \xi_{n}}^{\prime},
$$

otherwise

$$
\eta_{p_{\bullet}(\theta), \alpha}=\eta_{p_{0}(\theta), \alpha} \quad\left(\text { if } \alpha \in U_{\theta}^{*}\right) .
$$

Observe that as $\eta_{\varrho_{n}, \xi_{n}}^{\prime}$ was a cofinal branch in $\left(T_{p_{\bullet}\left(\varrho_{n}\right)}\right)_{<\delta_{\bullet}+1}=\left(T_{p_{0}^{\prime}\left(\varrho_{n}\right)}\right)_{<\delta_{\bullet}+1}$

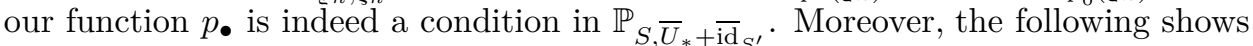
that $\forall n \in \omega p_{\bullet} \leq q_{n}$. Fix $n \in \omega$, then using $\mathbf{⿴ 囗 十}_{4} \sqrt{(d)}$ we have $q_{n} \uparrow\left(\bar{U}_{*}+\bar{W}_{n}\right) \in \mathbf{G}_{n}$, i.e. there exist $l_{0}, l_{1}, \ldots l_{n} \in \omega$, such that $\bigwedge_{j=0}^{n} p_{j, l_{j}} \leq_{\mathbb{P}} q_{n} \uparrow\left(\bar{U}_{*}+\bar{W}_{n}\right)$. This means that

$$
\bigwedge_{j=0}^{n} p_{j, l_{j}} \leq q_{n} \uparrow\left(\bar{U}_{*}+\bar{W}_{0}\right)=q_{n} \uparrow\left(\bar{U}_{*}\right),
$$

and

$$
\text { for each } 0<j \leq n \quad \eta_{q_{n}\left(\varrho_{j}\right), \xi_{j}} \subseteq \eta_{p_{j, l_{j}}\left(\varrho_{j}\right), \xi_{j}} \subseteq \eta_{\varrho_{j}, \xi_{j}}^{\prime}=\eta_{p_{\bullet}\left(\varrho_{j}\right), \xi_{j}} \text {. }
$$

On the other hand, for $j>n$ we have (recalling $\bar{q}=\left\langle q_{n}: n \in \omega\right\rangle$ is $\leq_{\mathbb{P}}$-decreasing by $⿴ 囗 十)$ that

therefore $p_{\bullet} \leq q_{n}$, indeed.

$$
\eta_{q_{n}\left(\varrho_{j}\right), \xi_{j}} \subseteq \eta_{q_{j}\left(\varrho_{j}\right), \xi_{j}} \subseteq \eta_{\varrho_{j}, \xi_{j}}^{\prime}=\eta_{p_{\bullet}\left(\varrho_{j}\right), \xi_{j}},
$$

Now assuming $p_{\bullet} \in \mathbf{G}_{S, \bar{U}_{*}+\overline{i d}_{S^{\prime}}}$ will easily yield a contradiction: First recall that $p_{*}$ (and therefore as well $q_{0}$ and $p_{\bullet}$ ) forced that $\dot{b}$ is a branch through $\dot{T}$. Then 田2 $\sqrt{(c)}$ implies that $p_{0}^{\prime}$, thus $p_{\bullet}$ as well determines $\dot{T}_{\leq \delta}$, and $p_{\bullet}$ forces (by 田4 $\sqrt{(e)}$ ) that each element of the $\delta_{\bullet}$ 'th level of $\dot{T}$ is the upper bound of $B_{i}$ for some $i \in \omega$. This means that

while at the same time

$$
p_{\bullet} \Vdash(\exists i \in \omega) \dot{b} \cap \dot{T}_{<\delta \bullet}=B_{i},
$$

$$
\left(q_{i} \wedge p_{0}^{\prime}\right) \Vdash \dot{b} \neq B_{i}
$$

since (3.9) holds.

This together with $p_{\bullet} \leq q_{i}, p_{0}^{\prime}$ gives the contradiction. Now we can turn to the proof of the claim.

Proof. (Claim 3.27)

For the construction of each sequence $\bar{p}_{n}$ and each $q_{n}$ we will work in $N_{1}$. This will need a lot of preparation.

Recall that $X \subseteq N_{0}$ denoted the indices of branches added by forcing with

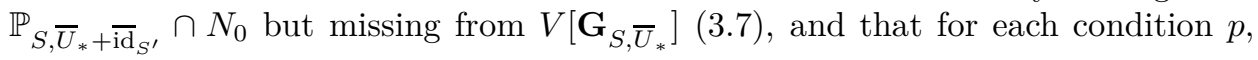


$\theta \in S_{\bullet}$, and $\delta<\omega_{1}$ the $\delta^{\prime}$ th level of $T_{p(\theta)}$ is (a subset of) $[\omega \cdot \delta, \omega \cdot(\delta+1)$ ). Define $E \subseteq N_{0}$ as follows.

$$
\begin{aligned}
e \in E \text { iff } & e \in N_{0}, \text { and } e=\left(u_{e}, \bar{\eta}_{e}\right), \text { where } u_{e} \in[X] \leq \omega, \\
& \bar{\eta}_{e}=\left\langle\eta_{e, \theta, \alpha}:\langle\theta, \alpha\rangle \in u_{e}\right\rangle, \text { such that } \\
& \eta_{e, \theta, \alpha} \subseteq \omega \cdot\left(\delta_{\theta, \alpha}+1\right) \text { for some } \delta_{\theta, \alpha}<\omega_{1}
\end{aligned}
$$

Definition 3.28. For each $n, p \in \mathbb{P}_{S, \bar{U}_{*}+\bar{W}_{n}}$, and $e \in E$, if for each $\langle\theta, \alpha\rangle \in u_{e}$ we have $\theta \in \operatorname{dom}(p)$, and for each $i<n\left\langle\varrho_{i}, \xi_{i}\right\rangle \notin u_{e}$ holds then define $p^{\frown e}$ as

$$
\begin{aligned}
& \operatorname{dom}\left(p^{\frown} e\right)=\operatorname{dom}(p), \\
& \left.u_{\left(p^{\frown} e\right)(\theta)}=u_{p(\theta)} \cup\left\{\alpha:\langle\theta, \alpha\rangle \in u_{e}\right\}\right) \quad\left(\forall \theta \in \operatorname{dom}\left(p^{\frown} e\right)\right), \\
& \eta_{\left(p^{\frown} e\right)(\theta), \alpha}= \begin{cases}\eta_{p(\theta), \alpha}, & \text { if } \alpha \in u_{p(\theta)}, \\
\eta_{e, \theta, \alpha}, & \text { if }\langle\theta, \alpha\rangle \in u_{e},\end{cases} \\
& \text { if this is a condition in } \mathbb{P}\left(\text { i.e. for each }\langle\theta, \alpha\rangle \in u_{e}\right. \\
& \eta_{e, \theta, \alpha} \text { is a cofinal branch of }\left(T_{p(\theta)}\right)_{<\delta+1} \text { for some } \delta \leq h t\left(T_{p(\theta)}\right), \\
& \text { otherwise } p^{\frown e=\emptyset .}
\end{aligned}
$$

Let $\mathcal{D}$ denote the set of dense subsets of $\mathbb{P}_{S, \bar{U}_{*}+\overline{\mathrm{id}}_{S^{\prime}}}$. Fix an enumeration

$$
\left\langle\left\langle J_{i}, \varepsilon_{i}\right\rangle: i \in \omega\right\rangle \in N_{1} \text { of }\left(\mathcal{D} \cap N_{0}\right) \times E,
$$

and let $k(D, e)$ denote the index of the pair $\langle D, e\rangle$ (i.e.

$$
\left.J_{k(D, e)}=D, \varepsilon_{k(D, e)}=e\right) \text {, then we also have } k \in N_{1} \text {, of course. }
$$

Fix a function $g \in N_{0}$

$$
\begin{aligned}
& g: \quad \mathbb{P}_{S, \bar{U}_{*}+\overline{\mathrm{id}}_{S^{\prime}}} \times \mathcal{D} \rightarrow \mathbb{P}_{S, \bar{U}_{*}+\overline{\mathrm{id}}_{S^{\prime}}} \\
& \text { with } \forall p, D \text { : } \\
& \text { - } g(p, D) \in D \text {, } \\
& \text { - } g(p, D) \leq p \text {, }
\end{aligned}
$$

(Then $g \in N_{0}$ obviously implies $\left(p, D \in N_{0} \Rightarrow g(p, D) \in N_{0}\right)$.)

We will have to define also the auxiliary sequence $\bar{r}=\left\langle r_{l}: l \in \omega\right\rangle$ with the following property:

$$
\begin{aligned}
& \circledast_{1} \bar{r} \in N_{1}, \\
& \circledast_{2} \text { for each } l r_{l} \in \mathbb{P}_{S, \bar{U}_{*}} \cap N_{0}, \\
& \circledast_{3} \text { for each } l p_{0, l+1} \leq r_{l} \leq p_{0, l}, \\
& \circledast_{4} \text { if there exists } p \in \mathbb{P}_{S, \bar{U}^{*}} \text { such that } p \leq p_{0, l} \text {, and } p \frown \varepsilon_{l} \text { is a condition } \\
& \quad \text { extending } p_{0, l} \text { in } \mathbb{P}_{S, \bar{U}^{*}+\overline{\mathrm{id}}_{S^{\prime}}} \text {, then } r_{l} \text { is such that. }
\end{aligned}
$$

Now we can construct the $p_{0, i}$ 's (and $r_{i}$ 's). Let $p_{0,0}=p_{*} \uparrow \bar{U}_{*}$. For obtaining the $p_{0, l}$ 's proceed as follows. Assume we have defined $p_{0,0}, p_{0,1}, \ldots, p_{0, l-1}$ (and as well the $r_{i}$ 's for $i<l-1$ ). Now if there exists $p \in \mathbb{P}_{S, \bar{U}^{*}} p \leq p_{0, l-1}$, s.t. $p^{\frown} \varepsilon_{l-1} \neq \emptyset$ but a condition extending $p_{0, l-1}$, then let $r_{l-1} \in N_{0}$ be such a $p$ (recall that $\varepsilon_{l-1} \in E \subseteq N_{0}$ by (3.13)), otherwise define $r_{l-1}=p_{0, l}=p_{0, l-1}$. Lastly, in the former case define $p_{0, l}=g\left(r_{l-1}, D_{l-1}\right) \uparrow \bar{U}_{*}$. It is clear from the construction and the definition of $g$ that $p_{0, l-1} \leq r_{l-1} \leq p_{0, l}$, and $r_{l-1}, p_{0, l} \in N_{0}$, and since every object as well as the series $\left\langle\varepsilon_{i}: i \in \omega\right\rangle$ are elements of $N_{1}$, we obtain $\bar{p}_{0}, \bar{r}_{0} \in N_{1}$, too. 
Finally, it is straightforward to check that the filter $\mathbf{G}_{0}$ generated by the $p_{0, l}$ 's meets every dense subset $D \in N_{0}$ of $\mathbb{P}_{S, \bar{U}_{*}}$. Fixing such a $D$

$$
D^{\prime}=\left\{p \in \mathbb{P}_{S, \bar{U}_{*}+\overline{\mathrm{id}}_{S^{\prime}}}: p\left\lceil\bar{U}_{*} \in D\right\}\right.
$$

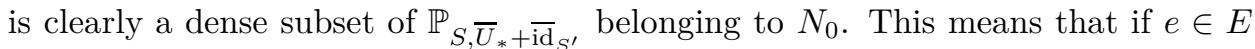
is the empty sequence, then there exists $i \in \omega$, such that $J_{i}=D^{\prime}$, and $\varepsilon_{i}=e$, therefore $p_{0, i+1} \in D$.

For $p_{0}^{\prime}$, first consider the condition $p_{0}^{\prime \prime} \in N_{1}$ consisting of only the generic trees given by $\mathbf{G}_{0}$ (for each $\theta \in \operatorname{dom}\left(p_{0}^{\prime \prime}\right)=N_{0} \cap S$ the tree $T_{p_{1}^{\prime}(\theta)}=\cup\left\{T_{p(\theta)}: p \in \mathbf{G}_{0}\right\}$

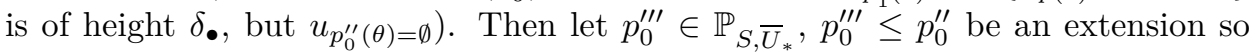
that for each $\theta \in S^{\prime} \cap N_{0}$ the tree $T_{p_{2}^{\prime}}(\theta)$ satisfies that for each branch $B$ through $\left(T_{p_{0}^{\prime \prime \prime}}(\theta)\right)_{<\delta_{\bullet}}=T_{p_{0}^{\prime \prime}(\theta)}$, if $B \in N_{1}$, then there is an upper bound of $B$ in $T_{p_{0}^{\prime \prime \prime}(\theta)}$. This can be done since $N_{1}$ is countable. Moreover, we choose the other part of $p_{0}^{\prime \prime \prime}$ so that for each $\theta, \alpha \in N_{0}$, if $\alpha \in U_{\theta}^{*}$ the chain $\eta_{p_{0}^{\prime \prime \prime}(\theta), \alpha}$ (with a top element) contains the chain $\cup\left\{\eta_{p(\theta), \alpha}: p \in \mathbf{G}_{0}\right\}$ which is given by $\mathbf{G}_{0}$ at this coordinate. This can be done as $\cup\left\{\eta_{p(\theta), \alpha}: p \in \mathbf{G}_{0}\right\} \in N_{1}$, since $\mathbf{G}_{0}, \bar{p}_{0} \in N_{1}$. Then clearly $p_{0}^{\prime \prime \prime} \leq p_{0, l}$ for each $l \in \omega$.

Finally, for the last item of $\mathbb{A}_{2}$ first recall that $\mathbb{P}_{S, \bar{U}_{*}}$ is an $\omega_{1}$-closed dense subposet of $\mathbb{P}_{S, \bar{U}_{*}}$ by Remark 3.11. Then if a countable increasing sequence in $\mathbb{P}_{S, \bar{U}_{*}^{*}}$ (where a first element stronger than $p_{0}^{\prime \prime \prime}$ ) decides more and more about the $\delta_{\bullet}$ 'th level of $\dot{T}$, then choosing $p_{0}^{\prime}$ to be an upper bound will work (e.g. choose an enumeration $\left\langle\dot{t}_{i}: i \in \omega\right\}$ of the $\delta_{\bullet}$ 'th level of $\dot{T}$, let $\left\langle s_{i}: i \in \omega\right\rangle$ enumerate $\dot{T}_{<\delta}$ in type $\omega$, and let $r_{j}$ decide whether the $j^{\prime}$ th ordered pair in the countable set $\left\{s_{i}: i \in \omega\right\} \times\left\{\dot{t}_{i}: i \in \omega\right\}$ is in $\left.\leq_{\dot{T}}\right)$.

The next step is to construct the $\bar{p}_{i}$ 's $(i>0)$ and the $q_{n}$ 's. This will be done simultaneously by induction. The induction is carried out in $V$, but each step can be done in $N_{1}$, which will guarantee that each $\bar{p}_{n} \in N_{1}$.

It is straightforward to check that choosing $q_{0}=p_{*}$ would satisfy our requirements, as e.g. $p_{0,0}=p_{*}\left\lceil\bar{U}_{*}\right.$. Then fixing $n>0$, and assuming that $\bar{p}_{i}, q_{i}$ are constructed for each $i<n$, first we construct $q_{n}$. Recall that $q_{n-1}\left\lceil\left(\bar{U}_{*}+\bar{W}_{n-1}\right) \in\right.$ $\mathbf{G}_{n-1}$ (by 田4 $(d)$ ).

Recall the definition of the set $E$ (3.13), and let

$$
E_{n-1}=\left\{e \in E: \forall i<n\left\langle\varrho_{i}, \xi_{i}\right\rangle \notin e\right\} .
$$

Using that $p_{*} \in \mathbb{P}_{S, \bar{U}_{*}+\overline{\mathrm{id}}_{S^{\prime}}}$ forced that $\dot{b}$ is not an element of $V\left[G_{S, \bar{U}_{*}+\bar{W}_{n-1}}\right]$, i.e. there is no $\mathbb{P}_{S, \bar{U}_{*}+\bar{W}_{n-1}}$-name of it, we argue that

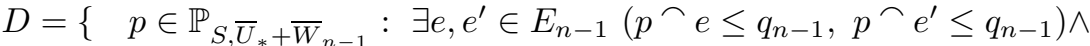

$$
\begin{aligned}
& \left.\left(\exists \delta<\omega_{1}, t \neq t^{\prime} \in \dot{T}_{\leq \delta} \backslash \dot{T}_{<\delta}: \quad(p \frown e \Vdash t \in \dot{b}) \wedge\left(p \frown e^{\prime} \Vdash t^{\prime} \in \dot{b}\right)\right)\right\}
\end{aligned}
$$

is dense in $\mathbb{P}_{S, \bar{U}_{*}+\bar{W}_{n-1}}$ under $q_{n-1} \uparrow\left(\bar{U}_{*}+\bar{W}_{n-1}\right)$. Indeed, assume on the contrary

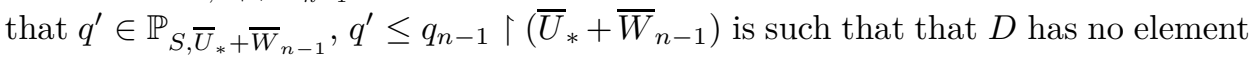
under $q^{\prime}$. Now for every $\delta<\omega_{1}$, consider the set

$$
\begin{aligned}
& D_{\delta}=\left\{\quad p \in \mathbb{P}_{S, \bar{U}_{*}+\bar{W}_{n-1}}:\left(p \leq q^{\prime}\right) \wedge\left(\exists e \in E_{n-1}:\left[p \frown e \leq q_{n-1}\right] \wedge,\right.\right. \\
& \left.\left.\wedge\left[\exists t_{p, e, \delta} \in \dot{T}_{\leq \delta} \backslash \dot{T}_{<\delta}: p \frown e \Vdash t_{p, e, \delta} \in \dot{b}\right]\right)\right\}
\end{aligned}
$$




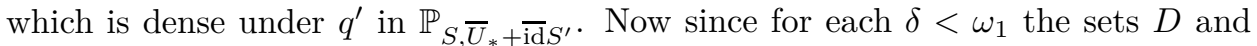
$D_{\delta}$ are disjoint, for $p \in D_{\delta}$ the witnessing $t_{p, e, \delta}$ doesn't depend on $e$, therefore $q^{\prime} \wedge q_{n-1}$ forces that $\dot{b}$ is in $V\left[\mathbf{G}_{S, \bar{U}_{*}+\bar{W}_{n-1}}\right]$ (i.e. forces that the $\mathbb{P}_{S, \bar{U}_{*}+\bar{W}_{n-1}}$-name $\left\{\left\langle p, t_{p, \delta}\right\rangle: \quad p \in D_{\delta}, \delta<\omega_{1}\right\}$ and $\dot{b}$ are equal).

Then as our set $D \in N_{0}$ is indeed dense we have that there exists a condition $q^{\prime \prime} \in \mathbf{G}_{n-1} \cap D$, witnessed by $t \neq t^{\prime}$ and $e, e^{\prime}$. Finally, if $t \in B_{n}$ then define $q_{n}=q^{\prime \prime} \frown e^{\prime}$, otherwise we can let $q_{n}=q^{\prime \prime} \frown e$, which are both stronger conditions than $q_{n-1}$ by the definition of $D$. It is straightforward to check 田4

As $q_{n}$ is already defined (and so are $\bar{p}_{i}, q_{i}$ for each $i<n$ ), we turn to the definition of $\bar{p}_{n}$, which we will do similarly to that of $\bar{p}_{0}$. Let $p_{n, 0}=q_{n}\left\lceil\left(\bar{U}_{*}+\bar{w}_{n}\right)\right.$, assume that $p_{n, 0}, p_{n, 1}, \ldots, p_{n, l-1}$ are already chosen.

If $\varepsilon_{l-1} \notin E_{n-1}$, then $p_{n, l}=p_{n, l-1}$, otherwise proceed as follows. Choose the sequence $\bar{e}=\bar{e}(n, l-1)=\left\langle e_{i}: \quad 1 \leq i \leq n+1\right\rangle \in E^{n+1 \backslash\{0\}}$ and the sequence $\bar{m}=\bar{m}(n, l-1)=\left\langle m_{i}: i \leq n\right\rangle \in \omega^{n+1}$ with the property

1) $e_{n+1}=\varepsilon_{l-1}$ and $m_{n}=l-1$,

2) for each $i<n+1$

$$
J_{m_{i}}=D \wedge " e_{i}=\left(e_{i+1} \text { plus }\left(\eta_{p_{i, m_{i}}\left(\varrho_{i}\right), \xi_{i}} \text { attained on }\left\langle\varrho_{i}, \xi_{i}\right\rangle\right)\right) " .
$$

Provided that the $e_{j}$ 's are defined for $j>i$, and as well each $m_{j}$ for $j \geq i$, let $e_{i} \in E$ be the element with $u_{e_{i}}=u_{e_{i+1}} \cup\left\{\left\langle\varrho_{i}, \xi_{i}\right\rangle\right\}, \bar{\eta}_{e_{i}} \supseteq \bar{\eta}_{e_{i+1}}, \eta_{e, \varrho_{i}, \xi_{i}}=\eta_{p_{i, m_{i}}\left(\varrho_{i}\right), \xi_{i}}$, and let $m_{i-1}=k\left(D, e_{i}\right)$. Observe that by our procedure, and by the definition of the function $k$ (3.15) we have $e_{1}=\varepsilon_{m_{0}}$, and also

$$
\eta_{e_{1}, \varrho_{n}, \xi_{n}}=\eta_{p_{n, l-1}\left(\varrho_{n}\right), \xi_{n}} \text {. }
$$

At some point later we will use the following fact, hence it is worth to note that for each $i, 1 \leq i \leq n$

$$
\bar{e}\left(i, m_{i}\right) \subseteq \bar{e}(n, l-1), \text { and } \bar{m}\left(i, m_{i}\right) \subseteq \bar{m}(n, l-1) .
$$

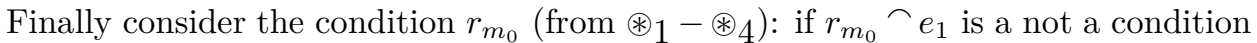
in $\mathbb{P}_{S, \bar{U}_{*}+\mathrm{id} \mid S^{\prime}}$, then let $p_{n, l}=p_{n, l-1}$, otherwise first define the auxiliary condition

$$
r_{\bullet}=g\left(r_{m_{0}} \frown e_{1}, D\right)
$$

and note that in this case $\eta_{\left(r_{m_{0}}\right.} \wedge_{\left.e_{1}\right)\left(\varrho_{n}\right), \xi_{n}}=\eta_{p_{n, l-1}\left(\varrho_{n}\right), \xi_{n}}$ by (3.18), therefore by the properties of $g$ we obtain

$$
\eta_{r_{\bullet}\left(\varrho_{n}\right), \xi_{n}} \supseteq \eta_{p_{n, l-1}\left(\varrho_{n}\right), \xi_{n}} .
$$

Recall that $p_{n, l-1}\left\lceil\bar{U}_{*} \in \mathbf{G}_{0}\right.$ by our induction hypotheses $\mathbb{A}_{3}$, and it can be seen from the construction of $p_{0, j}$ 's that in this case $p_{0, m_{0}+1}=r_{\bullet} \uparrow \bar{U}_{*} \in \mathbf{G}_{0}$. Therefore by (3.21) we have that $\left(r_{\bullet} \mid \bar{U}_{*}+\bar{w}_{n}\right) \wedge p_{n, l-1}$ is a condition in $\mathbb{P}_{\bar{U}_{*}+\bar{w}_{n}}$, and let

$$
p_{n, l}=\left(r_{\bullet} \mid \bar{U}_{*}+\bar{w}_{n}\right) \wedge p_{n, l-1} .
$$

Then clearly $p_{n, l} \leq p_{n, l-1}$, and $p_{n, l}\left\lceil\bar{U}_{*} \in \mathbf{G}_{0}\right.$. From $\mathbb{A}_{3}$ it only remained to check that $(d)$ and $(e)$ also hold. Since the whole construction of $\bar{p}_{n}$ took place in $N_{1}\left(k \in N_{1}\right.$ and so is the enumeration $\left.\left\langle\left\langle J_{i}, \varepsilon_{i}\right\rangle: i \in \omega\right\rangle, g \in N_{0}\right), \bar{p}_{n} \in N_{1}$ obviously

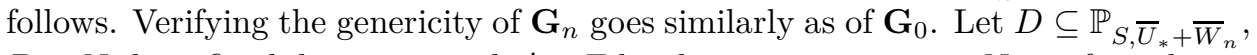
$D \in N_{0}$ be a fixed dense set, and $e^{\prime} \in E$ be the empty sequence. Now, if we choose

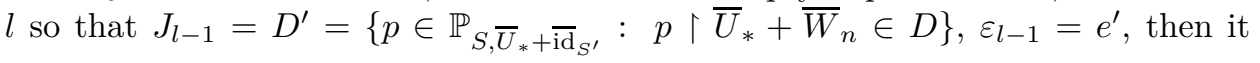


follows from the construction of $p_{k, j}$ 's, that of $\bar{m}=\bar{m}(n, l-1)$ and $\bar{e}=\bar{e}(n, l-1)$, and from (3.19) that

$$
p_{i, m_{i}+1}=\left(r_{\bullet} \mid \bar{U}_{*}+\bar{w}_{i}\right) \wedge p_{i, m_{i}} \text { if } 1 \leq i \leq n,
$$

and

therefore

$$
p_{0, m_{0}+1}=g\left(r_{m_{0}} \frown e_{1}\right) \uparrow \bar{U}_{*},
$$

$$
\bigwedge_{i \leq n} p_{i, m_{i}} \leq g\left(r_{m_{0}} \frown e_{1}\right) \uparrow\left(\bar{U}_{*}+\overline{W_{n}}\right) \in D^{\prime}
$$

$\square$ (Claim 3.27)

$\square$ (Lemma 3.26)

Lemma 3.29. Let $T \in V\left[\mathbf{G}_{S, \bar{U}_{*}}\right]$ be a Kurepa tree, $S^{\prime} \subseteq S \cap S$. $\left(S^{\prime} \in\right.$ $V), \quad \mathbf{G}_{\overline{\mathrm{id}}_{S^{\prime}}-\bar{U}_{*}}^{\circ} \subseteq \mathbb{P}_{\overline{\mathrm{id}}_{S^{\prime}}-\bar{U}_{*}}^{\circ}$ be generic over $V\left[\mathbf{G}_{S, \bar{U}_{*}}\right]$. Suppose that $b \in$ $V\left[\mathbf{G}_{S, \bar{U}_{*}},\left[\mathbf{G}_{S^{\prime},\left(\overline{\mathrm{id}}_{S^{\prime}}-\bar{U}_{*}\right)}^{\circ}\right] \backslash V\left[\mathbf{G}_{S, \bar{U}_{*}}\right]\right.$ is a new branch of $T$, and suppose that $\gamma \geq \kappa$ is a cardinal, and for each $\theta \in S^{\prime}$ the inequality $\left|\theta \backslash U_{\theta}^{*}\right| \geq \gamma$ holds. Then the filter $\mathbf{G}_{\overline{\mathrm{id}}_{S^{\prime}}-\bar{U}_{*}}$ adds at least $|\gamma|$-many new branches to $T$.

Proof. W.l.o.g. we can assume that $T \subseteq \omega_{1}$, and $\lambda$ is a cardinal (in $V\left[\mathbf{G}_{S, \bar{U}_{*}}\right]$ ). First we will choose a system $\bar{W}_{0}=\left\langle W_{0, \theta}: \theta \in S^{\prime}\right\rangle \in \prod_{\theta \in S^{\prime}} \mathcal{P}(\theta)$ with $\left(\forall \theta \in S^{\prime}\right)$ $\left|W_{0, \theta}\right|<\kappa$, and $b \in V\left[\mathbf{G}_{S, \bar{U}_{*}}\right]\left[\mathbf{G}_{\bar{W}_{0}}^{\circ}\right]:$ since $b \in V\left[\mathbf{G}_{S, \bar{U}_{*}}\right]\left[\mathbf{G}_{\overline{\mathrm{id}}_{S^{\prime}}^{\circ}-\bar{U}_{*}}^{\circ}\right], S^{\prime} \in V$ we can use Lemma 3.20 and obtain that $b \in V\left[\mathbf{G}_{S, \bar{U}_{*}}\right]\left[\mathbf{G}_{\overline{\mathrm{id}}_{S^{\prime}}-\bar{U}_{*}}^{\circ}\right]=V\left[\mathbf{G}_{S, \bar{U}_{*}+\overline{\mathrm{id}}_{S^{\prime}}}\right]$. And because $b \subseteq \mathcal{H}\left(\omega_{1}\right)^{V}$, applying Lemma 3.24 with $S$, and $\bar{U}=\bar{U}_{*}+\overline{\mathrm{id}}_{S^{\prime}}$, there exists $S_{*} \subseteq S, \bar{W}_{*} \in \prod_{S_{*} \backslash S^{\prime}} \mathcal{P}\left(U_{\theta}\right) \times \prod_{\theta \in S_{*} \cap S^{\prime}} \mathcal{P}(\theta)$ with

$$
b \in V\left[\mathbf{G}_{S_{*}, \bar{W}_{*}}\right] \subseteq V\left[\mathbf{G}_{S, \bar{U}_{*}+\bar{W}_{*}}\right]=V\left[\mathbf{G}_{S, \bar{U}_{*}}\right]\left[\mathbf{G}_{\bar{W}_{*}-\bar{U}_{*}}^{\circ}\right],
$$

where $\left|S_{*}\right|<\kappa$, and $\left|W_{\theta}^{*}\right|<\kappa$ for each $\theta \in S_{*}$. Then fixing $\bar{W}_{0} \in \prod_{\theta \in S^{\prime}} \mathcal{P}(\theta)$ so that $W_{0, \theta}=W_{\theta}^{*} \backslash U_{\theta}^{*}$ if $\theta \in S_{*}$, and $W_{0, \theta}=\emptyset$ for $\theta \in S \backslash S_{*}$ has the required properties.

Now, as $\left|W_{0, \theta}\right|<\kappa \leq \gamma$, and $\gamma \leq\left|\theta \backslash U_{\theta}^{*}\right|$ for each $\theta \in S^{\prime}$ we can fix for each $\alpha<\gamma$ a system $\bar{W}_{\alpha}=\left\langle W_{\alpha, \theta}: \theta \in S^{\prime}\right\rangle \in \prod_{\theta \in S^{\prime}} \mathcal{P}\left(\theta \backslash U_{\theta}^{*}\right)$ such that for every $\theta \in S^{\prime}$

(i) $W_{\alpha, \theta} \cap W_{\beta, \theta}=\emptyset$ for every $\alpha<\beta<\gamma$,

(ii) $\left|W_{0, \theta}\right|=\left|W_{\alpha, \theta}\right|$ for each $\alpha<\gamma$.

For each $0<\alpha<\gamma$ define the bijections

$$
\pi_{\alpha}: \bigcup_{\theta \in S^{\prime}}\{\theta\} \times W_{0, \theta} \rightarrow \bigcup_{\theta \in S^{\prime}}\{\theta\} \times W_{\alpha, \theta}
$$

where $\pi_{\alpha}\left\lceil\{\theta\} \times W_{0, \theta}\right.$ is a bijection to $\{\theta\} \times W_{\alpha, \theta}$. Then clearly each $\pi_{\alpha}$ induces an automorphism $\hat{\pi}_{\alpha} \in V\left[\mathbf{G}_{S, \bar{U}_{*}}\right]$ of $\mathbb{P}_{\bar{W}_{0}}^{\circ}$ and $\mathbb{P}_{\bar{W}_{\alpha}}^{\circ}$. Moreover, $\hat{\pi}_{\alpha}$ induces a natural operation $\hat{\pi}_{\alpha}^{*}$ from the class of $\mathbb{P}_{\bar{W}_{0}}^{\circ}$-names to the class of $\mathbb{P}_{\bar{W}_{\alpha}}^{\circ}$-names. Now fix a $\mathbb{P}_{\bar{W}_{0}}^{\circ}$-name $\dot{b}_{0} \in V\left[\mathbf{G}_{S, \bar{U}_{*}}\right]$ for our new branch $b \in V\left[\mathbf{G}_{S, \bar{U}_{*}}\right]\left[\mathbf{G}_{\bar{W}_{0}}^{\circ}\right]$, and choose an element $p_{\bullet} \in \mathbb{P}_{\bar{W}_{0}}^{\circ}$ forcing that $\dot{b}_{0}$ is a new branch, i.e.

$$
V\left[\mathbf{G}_{S, \bar{U}_{*}}\right] \models p_{\bullet} \Vdash \dot{b}_{0} \in \mathcal{B}(T) \backslash \mathcal{B}^{V\left[\mathbf{G}_{S, \bar{U}_{*}}\right]}(T) .
$$


Let $\mathbb{P}_{\bullet}^{\circ}=\mathbb{P}_{\sum_{\alpha<\gamma}^{\circ} \bar{W}_{\alpha}}$, i.e. adding the branches $\bigcup_{\alpha \in \gamma} W_{\alpha, \theta}$ to $\underset{\sim}{\underset{\sim}{T} \theta}$ for each $\theta \in S^{\prime}$, which is of course equal to the countably supported product of $\mathbb{P}_{\bar{W}_{\alpha}}^{\circ}$ 's $(\alpha<\gamma)$, and let $\mathbf{G}_{\bullet}^{\circ}$ denote the generic filter $\mathbf{G}_{\overline{\mathrm{id}}_{S^{\prime}}-\bar{U}_{*}}^{\circ} \cap \mathbb{P}_{\bullet}^{\circ}$.

We will show that in $V\left[\mathbf{G}_{S, \bar{U}_{*}}\right]\left[\mathbf{G}_{\bullet}^{\circ}\right] \subseteq V\left[\mathbf{G}_{S, \bar{U}_{*}}\right]\left[\mathbf{G}_{\overline{\mathrm{id}}_{S^{\prime}}-\bar{U}_{*}}^{\circ}\right]$ there are at least $\gamma$-many new branches of $T$, i.e.

$$
\left|\mathcal{B}(T) \cap\left(V\left[\mathbf{G}_{S, \bar{U}_{*}}\right]\left[\mathbf{G}_{\bullet}^{\circ}\right] \backslash V\left[\mathbf{G}_{S, \bar{U}_{*}}\right]\right)\right| \geq \lambda,
$$

by arguing that

$$
\begin{aligned}
& \otimes_{1} \text { for any } \alpha<\gamma\left(\text { in } V\left[\mathbf{G}_{S, \bar{U}_{*}}\right]\right) \\
& \qquad \hat{\pi}_{\alpha}(p \bullet) \Vdash_{\mathbb{P}_{\bullet}^{\circ}} \hat{\pi}_{\alpha}^{*}\left(\dot{b}_{0}\right) \notin V\left[\mathbf{G}_{S, \bar{U}_{*}}\right]\left[\mathbf{G}_{\bullet}^{\circ},<\alpha\right. \\
& \left(\text { where } \mathbf{G}_{\bullet}^{\circ},<\alpha\right. \\
& \text { stands for } \mathbf{G}_{\bullet}^{\circ} \cap \mathbb{P}_{\left.\sum_{\beta<\alpha}^{\circ} \bar{W}_{\beta}\right) \text {, and }} \\
& \otimes_{2}\left|\left\{\alpha<\gamma: \hat{\pi}_{\alpha}(p \bullet) \in \mathbf{G}_{\bullet}^{\circ}\right\}\right|=\gamma .
\end{aligned}
$$

This will complete the proof of Lemma 3.29 .

First we will prove 82 , for which recall that we assumed that $\gamma$ is a cardinal, and choose a system of uncountable regular cardinals $\left\{\rho_{\beta}: \beta<\chi<\gamma\right\}$, and a partition $\left\langle I_{\beta}: \beta<\chi\right\rangle$ of $\gamma$ with $\operatorname{otp}\left(I_{\beta}\right)=\rho_{\beta}$ for each $\beta<\chi$ (i.e. $I_{\beta} \cap I_{\delta}=\emptyset$ for $\beta<\delta<\rho$, and $\left.\bigcup_{\beta<\rho} I_{\beta}=\gamma\right)$. Then it is enough to verify

$$
(\forall \beta<\chi) \quad\left|\left\{\alpha \in I_{\beta}: \hat{\pi}_{\alpha}\left(p_{\bullet}\right) \in \mathbf{G}_{\bullet}^{\circ}\right\}\right|=\rho_{\beta},
$$

which can be seen by a standard density argument: Fix $\beta<\varrho, \alpha \in I_{\beta}$, then it suffices to show that

$$
D_{\beta, \alpha}=\left\{p \in \mathbb{P}_{\bullet}^{\circ}: p \leq \hat{\pi}_{\delta}\left(p_{\bullet}\right) \text { for some } \delta>\alpha, \delta \in I_{\beta}\right\} \text { is dense, }
$$

which obviously holds by the regularity of the uncountable $\rho_{\beta}=\left|I_{\beta}\right|$ (since for $\delta \in I_{\beta}$ we have $\hat{\pi}_{\delta}\left(p_{\bullet}\right) \in \mathbb{P}_{\bar{W}_{\delta}}^{\circ}, \mathbb{P}_{\bullet}^{\circ}$ is the countably supported product of $\mathbb{P}_{\bar{W}_{\alpha}^{\circ}}^{\circ}$ 's $(\alpha<\gamma)$, and $\left.I_{\beta} \subseteq \gamma\right)$.

For $\otimes_{1}$ first consider $\mathbb{P}_{\bullet}^{\circ}$ as the product of $\mathbb{P}_{\sum_{\beta<\gamma, \beta \neq \alpha}^{\circ}} \bar{W}_{\beta}$ and $\mathbb{P}_{\bar{W}_{\alpha}}^{\circ}$. We will need the following claim.

Claim 3.30. For each $p \in \mathbb{P}_{\bar{W}_{\alpha}}^{\circ}, p \leq \hat{\pi}_{\alpha}\left(p_{\bullet}\right)$ there exist $q_{0}, q_{1} \in \mathbb{P}_{\bar{W}_{\alpha}}^{\circ} q_{0}, q_{1} \leq p$, and the incomparable elements $t_{0}, t_{1}$ of the tree $T$ such that

$$
V\left[\mathbf{G}_{S, \bar{U}_{*}}\right]\left[\mathbf{G}_{\bullet, \gamma \backslash\{\alpha\}}^{\circ}\right] \models \quad\left(q_{i} \Vdash_{\mathbb{P}_{\bar{W}_{\alpha}}^{\circ}} t_{i} \in \hat{\pi}_{\alpha}^{*}\left(\dot{b}_{0}\right)\right) \quad \text { for each } i \in\{0,1\},
$$

where $\mathbf{G}_{\bullet, \gamma \backslash\{\alpha\}}^{\circ}=\mathbf{G}_{\bullet}^{\circ} \cap \mathbb{P}_{\sum_{\beta<\gamma, \beta \neq \alpha}^{\circ}}^{\circ} \bar{W}_{\beta}$.

Before proving the claim we verify that $\otimes_{1}$ follows from it. In fact

$$
\hat{\pi}_{\alpha}\left(p_{\bullet}\right) \Vdash_{\mathbb{P}_{\bullet}} \hat{\pi}_{\alpha}^{*}\left(\dot{b}_{0}\right) \notin V\left[\mathbf{G}_{S, \bar{U}_{*}}\right]\left[\mathbf{G}_{\bullet, \gamma \backslash\{\alpha\}}^{\circ}\right] .
$$

Since $\mathbf{G}_{\bullet}^{\circ} \subseteq \mathbb{P}_{\bullet}^{\circ}$ is generic over $V\left[\mathbf{G}_{S, \bar{U}_{*}}\right]$, and $\mathbb{P}_{\bullet}^{\circ}$ can be identified with

$$
\left(\mathbb{P}_{\sum_{\beta<\gamma, \beta \neq \alpha}^{\circ}}^{\circ} \bar{W}_{\beta}\right) \times \mathbb{P}_{\bar{W}_{\alpha}}^{\circ},
$$

by [Kun13, Lemma V.1.1] $\mathbf{G}_{\bullet, \gamma \backslash\{\alpha\}}^{\circ}=\mathbf{G}_{\bullet}^{\circ} \cap \mathbb{P}_{\sum_{\beta<\gamma, \beta \neq \alpha}^{\circ}} \bar{W}_{\beta}$ is generic over $V\left[\mathbf{G}_{S, \bar{U}_{*}}\right]$, and $\mathbf{G}_{\bullet, \alpha}^{\circ}=\mathbf{G}_{\bullet}^{\circ} \cap \mathbb{P}_{\bar{W}_{\alpha}}^{\circ}$ is generic over $V\left[\mathbf{G}_{S, \bar{U}_{*}}\right]\left[\mathbf{G}_{\bullet, \gamma \backslash\{\alpha\}}^{\circ}\right]$. For each branch $c \in$ 


$$
\begin{aligned}
\left.V\left[\mathbf{G}_{S, \bar{U}_{*}}\right]\left[\mathbf{G}_{\bullet, \gamma \backslash\{\alpha\}}^{\circ}\right] \text { of } T \text { define (in } V\left[\mathbf{G}_{S, \bar{U}_{*}}\right]\left[\mathbf{G}_{\bullet, \gamma \backslash\{\alpha\}}^{\circ}\right]\right) \\
D_{c}=\left\{q \in \mathbb{P}_{\bar{W}_{\alpha}}^{\circ}: \exists t \in T \backslash c \text { such that } q \Vdash_{\mathbb{P}_{\bar{W}_{\alpha}}^{\circ}} t \in \hat{\pi}_{\alpha}^{*}\left(\dot{b}_{0}\right)\right\},
\end{aligned}
$$

which is dense under $\hat{\pi}_{\alpha}\left(p_{\bullet}\right)$ by Claim 3.30 since for a fixed $p \in \mathbb{P}_{\bar{W}_{\alpha}^{\circ}}$ at most one $t_{i}$ can be in the branch $c$.

Proof. (Claim 3.30) First we argue that the statement holds in $V\left[\mathbf{G}_{S, \bar{U}_{*}}\right]$, i.e. for each $p \in \mathbb{P}_{\bar{W}_{\alpha}}^{\circ}, p \leq \hat{\pi}_{\alpha}\left(p_{\bullet}\right)$ there exist $q_{0}, q_{1} \in \mathbb{P}_{\bar{W}_{\alpha}} q_{0}, q_{1} \leq p$, and the incomparable elements $t_{0}, t_{1}$ of the tree $T$ such that

$$
V\left[\mathbf{G}_{S, \bar{U}_{*}}\right] \models \quad\left(q_{i} \Vdash_{\mathbb{P}_{\bar{W}_{\alpha}}^{\circ}} t_{i} \in \hat{\pi}_{\alpha}^{*}\left(\dot{b}_{0}\right)\right) \quad \text { for each } i \in\{0,1\} .
$$

Now (3.22) implies that

$$
V\left[\mathbf{G}_{S, \bar{U}_{*}}\right] \models \quad \hat{\pi}_{\alpha}\left(p_{\bullet}\right) \Vdash_{\mathbb{P}_{\bar{W}_{\alpha}}} \hat{\pi}_{\alpha}^{*}\left(\dot{b}_{0}\right) \in\left(\mathcal{B}(T) \backslash \mathcal{B}^{V\left[\mathbf{G}_{S, \bar{U}_{*}}\right]}(T)\right)
$$

since $\dot{b}_{0} \in V\left[\mathbf{G}_{S, \bar{U}_{*}}\right]$ is a $\mathbb{P}_{\bar{W}_{0}}^{\circ}$-name and $T \in V\left[\mathbf{G}_{S, \bar{U}_{*}}\right]$. Suppose that $p \leq \hat{\pi}_{\alpha}(p \bullet)$ is a counterexample, but then for the set

$$
b^{\prime}=\left\{t \in T: \exists q \in \mathbb{P}_{\bar{W}_{\alpha}}^{\circ}, q \leq p \text { s.t. } q \Vdash t \in \hat{\pi}_{\alpha}^{*}\left(\dot{b}_{0}\right)\right\} \in V\left[\mathbf{G}_{S, \bar{U}_{*}}\right]
$$

we have $p \Vdash \hat{\pi}_{\alpha}^{*}\left(\dot{b}_{0}\right)=b^{\prime}$ ( since $\hat{\pi}_{\alpha}\left(p_{\bullet}\right)$ forced that $\hat{\pi}_{\alpha}^{*}\left(\dot{b}_{0}\right)$ is a cofinal branch in $T$ ), a contradiction. Finally, fixing $p \leq \hat{\pi}_{\alpha}(p \bullet)$, if $q_{0}, q_{1} \in \mathbb{P}_{\bar{W}_{\alpha}}^{\circ} q_{0}, q_{1} \leq p$, and the incomparable elements $t_{0}, t_{1} \in T$ are such that (3.24) holds, then

$$
V\left[\mathbf{G}_{S, \bar{U}_{*}}\right]\left[\mathbf{G}_{\bullet, \gamma \backslash\{\alpha\}}^{\circ}\right] \models \quad\left(q_{i} \Vdash_{\mathbb{P}_{\bar{W}_{\alpha}}^{\circ}} t_{i} \in \hat{\pi}_{\alpha}^{*}\left(\dot{b}_{0}\right)\right) \quad \text { for each } i \in\{0,1\},
$$

since if $q_{i} \in \mathbf{H} \subseteq \mathbb{P}_{\bar{W}_{\alpha}}^{\circ}$ is generic over $V\left[\mathbf{G}_{S, \bar{U}_{*}}\right]\left[\mathbf{G}_{\bullet, \gamma \backslash\{\alpha\}}^{\circ}\right]$, and $t_{i} \notin \hat{\pi}_{\alpha}^{*}\left(\dot{b}_{0}\right)[\mathbf{H}]$ (for some $i \in\{0,1\}$ ), then $\mathbf{H}$ is generic over $V\left[\mathbf{G}_{S, \bar{U}_{*}}\right]$ too, and the same holds in $V\left[\mathbf{G}_{S, \bar{U}_{*}}\right][\mathbf{H}]$.

It is left to argue why Lemma 3.26 and Lemma 3.29 complete the proof of Theorem 3.1 (and Theorem 3.4). Suppose that $T \in V[\mathbf{G}]$ is a Kurepa tree (where $\mathbf{G} \subseteq \mathbb{P}=\mathbb{P}_{S_{\bullet}^{+}, \overline{\mathrm{id}}_{S_{\bullet}^{+}}}$is generic), and assume on the contrary that $\left|\mathcal{B}^{V[\mathbf{G}]}(T)\right| \notin S_{\bullet}$. We can also assume that $T \subseteq \mathcal{H}\left(\omega_{1}\right)^{V}$, and by Lemma 3.24 there exists $S_{*} \subseteq S_{\bullet}^{+}$, $\left|S_{*}\right|<\kappa, \bar{W}_{*}=\left\langle W_{\theta}^{*}: \theta \in S_{*}\right\rangle \in \prod_{\theta \in S_{*}}[\theta]^{<\kappa}$ such that $T \in V\left[\mathbf{G}_{S_{*}, \bar{W}_{*}}\right]$. For estimating $\left(2^{\omega_{1}}\right)^{V\left[\mathbf{G}_{S_{*}, \bar{W}_{*}}\right]}$ first a straightforward calculation yields that $\left|\mathbb{P}_{S_{*}, \bar{W}_{*}}\right|<$ $\kappa$ : Since $\left|\mathbb{P}_{S_{*},\left\langle\emptyset: \theta \in S_{*}\right\rangle}\right|=\left(\left|S_{*}\right|\left|\omega_{1}\right|\right)^{\omega}$ which is either $\left(\omega_{1} \cdot \omega_{1}\right)^{\omega}=\omega_{1}<\omega_{2}$ (if $\kappa=\omega_{2}$, by $\mathbf{C H}$ ), or $\gamma^{\omega}<\kappa$ (for some $\gamma<\kappa$, if $\kappa$ is inaccessible). Thus recalling the definition of $\mathbb{Q}_{\theta, W_{\theta}^{*}}$ 's, the fact $\sum_{\theta \in S_{*}}\left|W_{\theta}^{*}\right|<\kappa$ as $\kappa$ is regular, and $\sup W_{\kappa}^{*}<\kappa$ (if $\kappa \in S_{*}$ ) we have the following (in both cases regardless of whether $\kappa=\left(\omega_{2}\right)^{V}$, or an inaccessible)

$$
\left|\mathbb{P}_{S_{*}, \bar{W}_{*}}\right|=\left|\mathbb{P}_{S_{*},\left\langle\emptyset: \theta \in S_{*}\right\rangle}\right| \cdot\left(\left(\omega_{1}\right) \cdot\left(\sum_{\theta \in S_{*} \backslash\{\kappa\}}\left|W_{\theta}^{*}\right|\right)\right)^{\omega} \cdot\left(\left|W_{\kappa}^{*}\right| \cdot \sup W_{\kappa}^{*}\right)^{\omega}<\kappa .
$$

At this point we have to discuss the two cases (i.e. whether $\kappa \in S_{\bullet}$ ) differently, arguing that in both cases there are branches outside $V\left[\mathbf{G}_{S_{*}, \bar{W}_{*}}\right]$.

If $\kappa=\omega_{2} \in S_{\bullet}$, then as

$$
V \models\left|\mathbb{P}_{S_{*}, \bar{W}_{*}}\right|^{\omega_{1} \cdot\left|\mathbb{P}_{S_{*}, \bar{W}_{*}}\right|}=\omega_{2},
$$


we have

$$
V\left[\mathbf{G}_{S_{*}, \bar{W}_{*}}\right] \models 2^{\omega_{1}}=\omega_{2},
$$

therefore as $\left|\mathcal{B}^{V[\mathbf{G}]}(T)\right| \notin S_{\bullet}$, there are branches of $T$ in $V[\mathbf{G}]$ not in $V\left[\mathbf{G}_{S_{*}, \bar{W}_{*}}\right]$. On the other hand, if $\kappa \notin S$ • is inaccessible, then we obtain that

$$
V\left[\mathbf{G}_{S_{*}, \bar{W}_{*}}\right] \models|\mathcal{B}(T)| \leq 2^{\omega_{1}}<\kappa
$$

and as $\kappa$ remains a cardinal in $V[\mathbf{G}]$ (by Claim 3.25 ), and

$$
V[\mathbf{G}] \models\left|\mathcal{B}(T) \cap V\left[\mathbf{G}_{S_{*}, \bar{W}_{*}}\right]\right|=\omega_{1},
$$

we conclude that this case there also must be branches of $T$ not in $V\left[\mathbf{G}_{S_{*}, \bar{W}_{*}}\right]$ as $T$ is a Kurepa tree in $V[\mathbf{G}]$. Now let $\bar{R} \in \prod_{\theta \in S_{\bullet}^{+} \backslash S_{\bullet}} \mathcal{P}(\theta), R_{\theta}=\theta \backslash W_{\theta}^{*}$, then

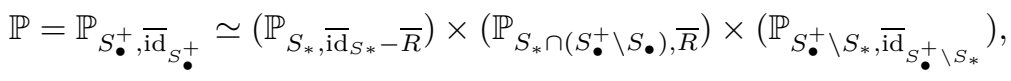

and there are no new sequences of type $\omega$ in $V[\mathbf{G}]$ (by Claim 3.23 ), and the second component is $\omega_{1}$-closed, the third component has an $\omega_{1}$-closed dense subset (which

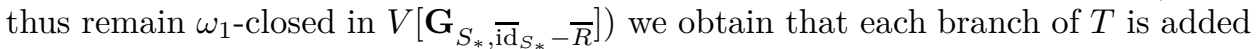

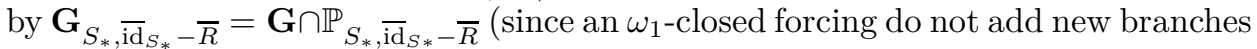
to Kurepa trees [Kun13, Lemma V.2.26]). We only have to derive a contradiction from

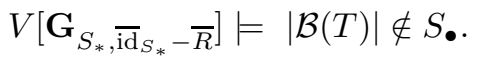

Now letting $\partial=\left|\mathcal{B}^{V\left[\mathbf{G}_{\left.S_{*}, \overline{\mathrm{id}}_{S_{*}}-\bar{R}\right]}\right.}(T)\right| \notin S_{\bullet}, S_{*}^{-}=S_{*} \cap S_{\bullet} \cap \partial, S_{*}^{+}=\left(S_{*} \cap S_{\bullet}\right) \backslash S_{*}^{-}$ by Lemma 3.20 we have

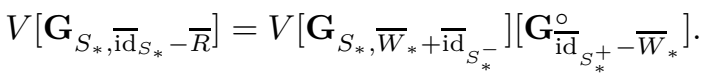

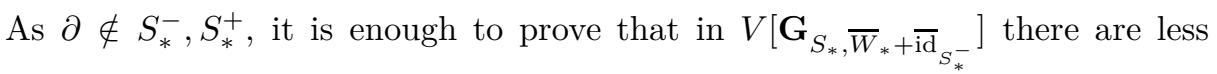
than $\partial$-many branches of $T$, because if $\mathbf{G}_{\overline{\mathrm{id}}_{S_{*}^{+}}}^{\circ} \bar{W}_{*}$ adds new branches, then adds $\min \left(S_{*}^{+}\right)$-many new branches by Lemma 3.29 (since each $\left|W_{\theta}^{*}\right|<\kappa \leq \min \left(S_{\bullet}\right) \leq$ $\left.\min \left(S_{*}^{+}\right)\right)$.

Now if $\partial=\kappa$, then $S_{*}^{-}=\emptyset$, we are done, so we can assume that $\partial>\kappa$, and $\sup S_{*}^{-} \geq \kappa$. As $\left|S_{*}\right|<\kappa($ in $V)$, and our conditions ( $\underline{\text { Case1 }} /($ iii) , or Case2 $/(i i)$ ) states that then $\sup \left(S_{*} \cap S_{\bullet} \cap \partial\right) \in S_{\bullet}$ implying $\sup S_{*}^{-}<\partial$. Therefore using that $W_{\theta}^{*} \subseteq \theta$ we get $\sum_{\theta \in S_{*}^{-}}\left|W_{\theta}^{*}\right| \leq\left|\sup S_{*}^{-}\right|^{2}<\partial$. Now by Lemma 3.26 for each branch

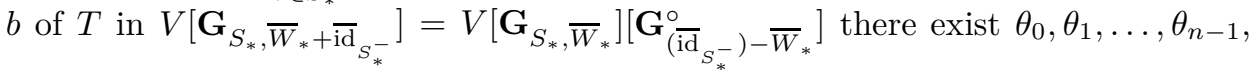
$U_{\theta_{0}}^{\bullet}, U_{\theta_{1}}^{\bullet}, \ldots, U_{\theta_{n-1}}^{\bullet}$ finite such that $b \in V\left[\mathbf{G}_{S_{*}, W_{*}}{ }^{*}\right]\left[\mathbf{G}_{\bar{U}_{\bullet}}^{\circ}\right]$. Therefore, as $\left|\mathbb{P}_{\bar{U}}^{\circ}\right|=$ $\omega_{1}^{n}=\omega_{1}$, counting the nice $\mathbb{P}_{\bar{U}}^{\circ}$-names of subsets $T$ for each possible $n$, sequence of $\theta$ 's, and $\bar{U}$.

$\mathcal{B}(T) \cap\left(V\left[\mathbf{G}_{S_{*}, \bar{W}_{*}}\right]\left[\mathbf{G}_{\left(\overline{\mathrm{id}}_{S_{*}^{-}}\right)-\bar{W}_{*}}^{\circ}\right] \backslash V\left[\mathbf{G}_{S_{*}, \bar{W}_{*}}\right]\right) \leq\left(\left|\sup S_{*}^{-}\right|<\omega \cdot \omega_{1}^{\omega_{1}}\right)^{V\left[\mathbf{G}_{S_{*}, \bar{W}_{*}}\right]} \leq \sup S_{*}^{-}$,

which is smaller than $\partial$, a contradiction.

For $V[\mathbf{G}] \models 2^{\omega_{1}}=\lambda$ we only need to show that $2^{\omega_{1}} \leq \lambda$. But a similar straightforward calculation yields that $\mathbb{P}=\mathbb{P}_{S_{\bullet}^{+}, \overline{\mathrm{id}}_{S_{\bullet}^{+}}}$is of cardinality $\lambda$, and then (using $\kappa$-cc and the equality $\lambda^{<\kappa}=\lambda$ ) by counting the possible nice names for subsets of $\omega_{1}$ we obtain the desired inequality. 
Remark 3.31. If $S_{\bullet}$ also satisfies

$$
\forall \mu \in S_{\bullet}: \operatorname{cf}(\mu)<\kappa \rightarrow \mu^{+} \in S_{\bullet},
$$

and GCH holds in $V$ then $S_{\bullet} \backslash\{\lambda\}$ is the spectrum for the Jech-Kunen trees in $V[\mathbf{G}]$. (A tree $T$ of height $\omega_{1}$ and power $\omega_{1}$ is a Jech-Kunen tree if $\omega_{1}<|\mathcal{B}(T)|<2^{\omega_{1}}$.) For more on Jech-Kunen trees see also JS93, JS92, JS94. Note that CH in the final model implies that the product of countably many Jech-Kunen trees is a Jech-Kunen tree, so is the diagonal product of $\omega_{1}$-many Jech Kunen trees, hence (3.25) cannot be dropped.

One can obtain similar cardinal arithmetic conditions for $\mathrm{Sp}_{\mu}$ with $\mu$ large.

\section{The NECESSity of THE INACCESSIBLE CARDINAL}

In this section we prove that if $\omega_{2}$ is not an element of the spectrum, then $\omega_{2}$ is inaccessible in $L$. The idea of using transitive collapses of elementary submodels of constructible sets as nodes of a tree goes back to Solovay's original unpublished argument for the consistency strength of the negation of the Kurepa Hypothesis. Although the next proof is deemed to be well-known, for the sake of completeness we include the proof as there is probably no known source to cite.

Theorem 4.1. Suppose that $\omega_{2}^{V}$ is a successor in L. Then there exists a Kurepa tree $T$ with $\mathcal{B}^{V}(T)=\omega_{2}$.

Proof. We will use an extension of $L$, an inner model between $L$ and $V$, what serves as the motivation for the following definition of relative constructibility, which can be found in e.g. Kan03].

Definition 4.2. For a set $A$ define $L[A]=\bigcup_{\alpha \in O N} L_{\alpha}[A]$ by transfinite recursion as follows. $L_{0}[A]=\emptyset, L_{\alpha+1}[A]=\operatorname{def}_{A}\left(L_{\alpha}[A]\right)$, and $\alpha$ limit $L_{\alpha}[A]=\bigcup_{\beta<\alpha} L_{\beta}[A]$ (where $\operatorname{def}_{Y}(X)$ are the subsets of $X$ that can be defined in the structure $(X, \in \uparrow$ $(X \times X), Y \cap X)$ by parameters from $X$, see Kan03, Chapter $1, \S 3]$.

The following is standard easy exercise, but for the sake of completeness we include the proof.

Claim 4.3. There exists a set $A \subseteq \omega_{1}$ such that $\omega_{1}^{L[A]}=\omega_{1}, \omega_{2}^{L[A]}=\omega_{2}$.

Proof. If $\omega_{2}^{V}=\left(\lambda^{+}\right)^{L}$, where $|\lambda|=\omega_{1}$, then in a single subset $A$ of $\omega_{1}$ we can code a well-ordering of $\omega_{1}$ in type $\lambda$, and also for each $\alpha<\omega_{1}$ a well-ordering of $\omega$ in type $\alpha$ in the obvious fashion, and such that $L$ can read this coding (implying $\left.\omega_{1}^{L[A]}=\omega_{1}, \omega_{2}^{L[A]}=\omega_{2}\right)$ : First let $\left\langle X_{\alpha}: \alpha \leq \omega_{1}\right\rangle \in L$ be a set of pairwise disjoint sets of $\omega_{1}$ with $\left|X_{\alpha}\right|^{L}=\omega$ for each $\alpha<\omega_{1}$, and $\left|X_{\omega_{1}}\right|^{L}=\omega_{1}$, then for each $\alpha<\omega_{1}$ we can code the well ordering $X_{\alpha}$ in order type $\alpha$, and the well ordering of $X_{\omega_{1}}$ in type $\lambda$ in a subset $A^{\prime}$ of $\bigcup_{\alpha \leq \omega_{1}} X_{\alpha}^{2} \subseteq \omega_{1}^{2}$. Finally, taking the preimage of this set under a bijection $f \in L$ between $\omega_{1}$ and $\omega_{1}^{2}$, i.e. $A=f^{-1}\left(A^{\prime}\right)$ works.

We have to recall a classical Lemma [Kan03, Theorem 3.3]. Recall that $\mathcal{L}_{\in}\left(R_{A}\right)$ stands for the (first-order) language of set theory extended by the unary predicate $R_{A}$.

Lemma 4.4. There is a sentence $\sigma \in \mathcal{L}_{\in}\left(R_{A}\right)$ such that for every transitive set $N$

$$
(N, \in, X \cap N) \models \sigma \text { implies } N=L_{\gamma}[X] \text { for some limit } \gamma \text {. }
$$


In particular, if $M \prec\left(L_{\beta}[X], \in, X \cap L_{\beta}[X]\right)$, where $\beta$ is a limit ordinal and $\pi$ is the collapsing isomorphism from $M$ onto the transitive set $\operatorname{ran}(\pi)$, then the Mostowski collapse

$$
\operatorname{ran}(\pi)=L_{\gamma}[\{\pi(x): x \in M \cap X\}]
$$

for some $\gamma \leq \beta$.

The following is immediate.

Claim 4.5. For each infinite ordinal $\beta$ and $Y \subseteq L_{\beta}[X]$, if $Y \in L[X]$ and $X \subseteq$ $L_{\beta}[X]$, then $\mu=\left(|\beta|^{+}\right)^{L[X]}$ implies $Y \in L_{\mu}[X]$.

(Working in $L[X]$, if $Y \in L_{\gamma}[X]$, then let $M \prec L_{\gamma}[X]$ with $\{Y\} \cup L_{\beta}[X] \subseteq M$, $|M|=\left|L_{\beta}[X]\right|$, and apply the lemma recalling that $\pi\left\lceil L_{\beta}[X]\right.$ is the identity.)

Now we can turn to the definition of the tree $T$, which will be defined by its branches.

Recall that there exists a definable well-order on $L[A]$, which is downward absolute to almost every initial segment of $L[A]$ (to the ones indexed by limit ordinals) Kan03, Theorem 3.3]:

Lemma 4.6. There exists a formula $\varphi \in \mathcal{L}_{\in}\left(R_{A}\right)$ (i.e. in the language of set theory extended with the unary relation symbol $A)$ which define a well-ordering on $(L[A], \in, A)$, moreover if $\delta$ is a limit ordinal, $x, y \in L_{\delta}[A]$, then

$$
(L[A], \in, A) \models \varphi(x, y) \quad \Longleftrightarrow \quad\left(L_{\delta}[A], \in, A \cap L_{\delta}[A]\right) \models \varphi(x, y) .
$$

From now on ' $x<_{L[A]} y$ ' abbreviates $\varphi(x, y)$.

We will take Skolem hulls many times, thus we need to introduce the following variant of this standard notion.

Definition 4.7. Let $(M, \in, X, \partial), M \subseteq L[A]$ be a set model of the language $\mathcal{L}_{\in}\left(R_{A}, c_{\partial}\right)$ with $\emptyset \in M, M^{\prime} \subseteq M$ such that the well-ordering formula $\varphi \in \mathcal{L}_{\in}\left(R_{A}\right)$ from Lemma 4.6 is absolute to $M$, i.e.

$$
(\forall x, y \in M):(L[A], \in, A) \models \varphi(x, y) \quad \text { iff }(M, \in, X) \models \varphi(x, y),
$$

e.g. when $(M, \in, X)=\left(L_{\zeta}[A], \in, A \cap L_{\zeta}[A]\right)$ for some limit ordinal $\zeta$. Then the Skolem-hull of $M^{\prime}$ in $(M, \in, X, \partial)$ (in symbols, $\left.\mathfrak{H}^{(M, \in, X, \partial)}\left(M^{\prime}\right)\right)$ is the closure of $M^{\prime}$ under the functions $f_{\psi}^{(M, \in, X, \partial)}$ for each formula $\psi\left(v_{0}, v_{1}, \ldots, v_{n_{\psi}}\right) \in \mathcal{L}_{\in}\left(R_{A}, c_{\partial}\right)$ with $n_{\psi}+1$ free variables, where the function $f_{\psi}^{(M, \in, X, \partial)}$ satisfies the following.

$$
f_{\psi}^{(M, \in, X, \partial)}: M^{n_{\psi}} \rightarrow M
$$

is defined so that for every $\left\langle x_{1}, x_{2}, \ldots, x_{n_{\psi}}\right\rangle \in M^{n_{\psi}}$ :

$$
\begin{gathered}
\text { if } \exists y ! \in M \text { s.t. }(M, \in, X, \partial) \models \psi\left(y, x_{1}, x_{2}, \ldots, x_{n_{\psi}}\right), \\
\text { then let } f_{\psi}^{(M, \in, X, \partial)}\left(x_{1}, x_{2}, \ldots, x_{n_{\psi}}\right) \text { be the unique such } y, \\
\text { otherwise let } f_{\psi}^{(M, \in, X, \partial)}\left(x_{1}, x_{2}, \ldots, x_{n_{\psi}}\right)=\emptyset .
\end{gathered}
$$

Then the fact that for each formula $\psi^{\prime}$ we can define the formula saying that $y$ is the least $y$ (w.r.t. the well-order given by $\varphi$ ) satisfying $\psi^{\prime}\left(y, x_{1}, x_{2}, \ldots x_{n_{\psi^{\prime}}}\right)$ together with the Tarski-Vaught criterion implies that the closure is an elementary submodel of $M$, in symbols, $M^{\prime} \prec(M, \in, X, \partial)$. 
Observe that this closure only depends on the isomorphism class of $(M, \in, X, \partial)$ by the absoluteness of the well-ordering formula $\varphi$ (4.1).

Choose $\xi<\omega_{2}$ such that

(4.2) $\xi$ is the minimal ordinal $\left(\forall \alpha<\omega_{1}\right) \exists f_{\alpha} \in L_{\xi}[A]$ bijection between $\omega$ and $\alpha$ (which can be done due to Corollary 4.5, in fact $\xi=\omega_{1}$, but we won't use this equality, hence we don't argue that).

Now we will define an operation which assigns for each $\delta \in\left[\xi, \omega_{2}\right)$ the ordinal $\delta^{\prime}<\omega_{2}$ in the following way. We would like to choose $\delta^{\prime}$ so that in $L_{\delta^{\prime}}[A]$ it is true that for each set $x$ there exists a surjection from $\omega_{1}$ to $x$, and for $\delta^{\prime \prime} \neq \delta^{\prime}$ the structures $\left(L_{\delta^{\prime}}[A], \in, A, \delta_{0}\right)$ and $\left(L_{\delta^{\prime \prime}}[A], \in, A, \delta_{0}\right)$ cannot be elementarily equivalent.

Definition 4.8. Fix $\delta \in\left[\xi, \omega_{2}\right)$, and define $\delta^{\prime}$ to be the least ordinal such that

a) $\delta \in L_{\delta^{\prime}}[A]$

b) for each $x \in L_{\delta^{\prime}}[A]$ there is a bijection $f \in L_{\delta^{\prime}}[A]$ between $\omega_{1}$ and $x$,

c) taking the sentence $\sigma$ from Lemma $4.4\left(L_{\delta^{\prime}}[A], \in, A\right) \models \sigma$.

(Using Claim 4.5 and $\left(\left|L_{\alpha}[A]\right|=|\alpha|\right)^{L[A]}$ for $\alpha \geq \omega$ it is easy to see that we can do this closure operation, and there is such a $\delta^{\prime}<\omega_{2}$.) Then we have

$$
\left(\delta^{\prime} \text { is a limit }\right) \bigwedge\left(L_{\delta^{\prime}}[A] \models \text { ' } \omega_{1} \text { is the largest cardinal') },\right.
$$

and also the desired uniqueness by our next claim.

Claim 4.9. There is a statement $\sigma^{\prime} \in \mathcal{L}_{\in}\left(R_{A}, c_{\partial}\right)$ such that for each $\delta \in\left[\xi, \omega_{2}\right)$ $\left(L_{\delta^{\prime}}[A], \in, A, \delta\right) \models \sigma^{\prime}$, moreover, for each $\delta>\omega_{1}$ and $\delta^{\prime \prime}>\delta$

$$
\left(\left(L_{\delta^{\prime \prime}}[A], \in, A, \delta\right) \models \sigma^{\prime}\right) \Rightarrow\left(\delta^{\prime \prime}=\delta^{\prime}\right) .
$$

Proof. First define $\sigma^{\prime \prime}=\sigma \wedge\left(\forall y \exists f: \omega_{1} \rightarrow y\right.$ bijection $)$ and let $\sigma^{\prime}$ be the following sentence

$$
\sigma^{\prime}=\sigma^{\prime \prime} \wedge\left(\neg(\exists X)(X \text { is transitive }) \wedge\left(\sigma^{\prime \prime}\right)^{X} \wedge(\delta \in X)\right)
$$

(where under $\psi^{X}$ we always mean the formula $\psi \in \mathcal{L}_{\in}\left(R_{A}, c_{\partial}\right)$ relativized to $X$, and $\sigma$ is from Lemma 4.4).

Now fix $\delta \in\left[\xi, \omega_{2}\right)$, and for each ordinal $0<\alpha<\omega_{1}$ define $M_{\delta, \alpha}$ to be the Skolem-hull

$$
M_{\delta, \alpha}=\mathfrak{H}^{\left(L_{\delta^{\prime}}[A], \in, A, \delta\right)}(\alpha) \quad\left(\text { for each } \alpha<\omega_{1}\right)
$$

Also define

$$
M_{\delta, 0}=\emptyset \text {. }
$$

Then

$$
\left.M_{\delta, \alpha} \prec\left(L_{\delta^{\prime}}[A], \in, A, \delta\right) \quad \text { (for each } \alpha>0\right) \text {. }
$$

Observe that whenever $M^{*} \prec\left(L_{\delta^{\prime}}[A], \in, A, \delta\right)$ we have for the Skolem functions from Definition 4.7 that $f_{\psi}^{\left(L_{\delta^{\prime}}[A], \in, A, \delta\right)} \uparrow\left(M^{*}\right)^{n_{\psi}}=f_{\psi}^{\left(M^{*}, \in, A \cap M^{*}, \delta\right)}$, hence

(4.7) $\forall M^{\prime} \subseteq M^{*} \prec\left(L_{\delta^{\prime}}[A], \in, A, \delta\right): \quad \mathfrak{H}^{\left(L_{\delta^{\prime}}[A], \in, A, \delta\right)}\left(M^{\prime}\right)=\mathfrak{H}^{\left(M^{*}, \in, A \cap M^{*}, \delta\right)}\left(M^{\prime}\right)$.

Now as we defined $\left\langle M_{\delta, \alpha}: \alpha<\omega_{1}\right\rangle$ note that

$$
\left(M \prec\left(L_{\delta^{\prime}}[A], \in, A, \delta\right)\right) \wedge(|M|=\omega) \rightarrow\left(M \cap \omega_{1} \in \omega_{1}\right),
$$

in particular

$$
M_{\delta, \alpha} \cap \omega_{1} \in \omega_{1}
$$


since (4.2) together with $\xi \leq \delta<\delta^{\prime}$ implies that in $L_{\delta^{\prime}}[A]$ there is an enumeration of each ordinal less than $\omega_{1}$ (and $M_{\delta, \alpha}$ is countable). This implies that

$$
\left(C_{\delta}=\left\{\alpha<\omega_{1}: M_{\delta, \alpha} \cap \omega_{1}=\alpha\right\} \text { is a club in } \omega_{1}\right) \wedge\left(0 \in C_{\delta}\right) .
$$

It is easy to see that

$$
\forall \alpha<\omega_{1}: M_{\delta, \alpha}=M_{\delta, \min \left(C_{\delta} \backslash \alpha\right)} .
$$

For later use we verify the following statement.

\section{Claim 4.10.}

$$
\bigcup_{\alpha<\omega_{1}} M_{\delta, \alpha}=L_{\delta^{\prime}}[A] .
$$

Proof. Since the union of an increasing chain of elementary submodels is an elementary submodel, we have $M_{\omega_{1}}=\bigcup_{\alpha<\omega_{1}} M_{\delta, \alpha} \prec\left(L_{\delta^{\prime}}[A], \in, A, \delta\right)$. Now recall, that in $L_{\delta^{\prime}}[A]$ every set $x$ admits a surjection from $\omega_{1}$ onto $x$, therefore $\omega_{1} \subseteq M_{\omega_{1}}$ implies that $M_{\omega_{1}}$ is transitive. Then by Lemma 4.4 and $M_{\omega_{1}} \models \sigma$ we have $M_{\omega_{1}}=$ $L_{\delta^{\prime \prime}}[A]$ for some $\delta^{\prime \prime}>\delta$. But then either $M_{\omega_{1}} \in L_{\delta^{\prime}}[A]$, or $M_{\omega_{1}}=L_{\delta^{\prime}}[A]$, and because the former would contradict Claim 4.9, we arrive at our conclusion.

For each $\alpha \in C_{\delta}$ and $\beta<\omega_{1}$, if $\alpha=\max \left(C_{\delta} \cap(\beta+1)\right)$, then let $N_{\delta, \beta, \alpha}$ be the range of the Mostowski-collapse $\pi_{\delta, \alpha}$ of $\left(M_{\delta, \alpha}, \in\right)$, and let $A_{\delta, \beta, \alpha}=\pi_{\delta, \alpha}(A)$, $\partial_{\delta, \beta, \alpha}=\pi_{\delta, \alpha}(\delta)$ :

$$
\pi_{\delta, \alpha}: M_{\delta, \alpha} \rightarrow N_{\delta, \beta, \alpha}
$$

which is of course not only an isomorphism between $\left(M_{\delta, \alpha}, \in\right)$ and $\left(N_{\delta, \beta, \alpha}, \in\right)$, but witnesses

$$
\left(M_{\delta, \alpha}, \in, A \cap M_{\delta, \alpha}, \delta\right) \simeq\left(N_{\delta, \beta, \alpha}, \in, A_{\delta, \beta, \alpha}, \partial_{\delta, \beta, \alpha}\right) .
$$

Now we are ready to construct the tree $T$. For a fixed $\delta \in\left[\xi, \omega_{2}\right), \alpha \in C_{\delta}$, $\beta<\omega_{1}$, if $0<\alpha=\max \left(C_{\delta} \cap(\beta+1)\right)$ holds then we define

$$
t_{\delta, \beta, \alpha}=\left(N_{\delta, \beta, \alpha}, \in, A_{\delta, \beta, \alpha}, \partial_{\delta, \beta, \alpha}\right)
$$

i.e. the structure $\left(N_{\delta, \beta, \alpha}, \in\right)$ extended by the one-place relation for the image of $A \in M_{\delta, \alpha}$ under the collapsing isomorphism, and the constant symbol for $\partial_{\delta, \beta, \alpha}$. For $\max \left(C_{\delta} \cap(\beta+1)\right)=0$ let $t_{\delta, \beta, 0}=\emptyset$.

Observe that given $t=t_{\delta, \beta, \alpha}$ we can decode $\alpha$ from $t$, as $\alpha$ is the first uncountable ordinal of $t$.

Definition 4.11. Define

$$
T=\left\{\left(\beta, t_{\delta, \beta, \alpha}\right): \delta \in\left[\xi, \omega_{2}\right), \beta<\omega_{1}, \alpha=\max \left(C_{\delta} \cap(\beta+1)\right)\right\},
$$

with the partial order $\left(\beta_{0}, t_{\delta_{0}, \beta_{0}, \alpha_{0}}\right) \leq_{T}\left(\beta_{1}, t_{\delta_{1}, \beta_{1}, \alpha_{1}}\right)$ iff either $\alpha_{0}=0$ (thus $t_{\delta_{0}, \beta_{0}, \alpha_{0}}$ is the empty structure), or

(i) $\beta_{0} \leq \beta_{1}$, and

(ii) taking the Skolem-hull $M$ of $\alpha_{0}$ in

$$
t_{\delta_{1}, \beta_{1}, \alpha_{1}}=\left(N_{\delta_{1}, \beta_{1}, \alpha_{1}}, \in, A_{\delta_{1}, \beta_{1}, \alpha_{1}}, \partial_{\delta_{1}, \beta_{1}, \alpha_{1}}\right)
$$

(i.e. $M=\mathfrak{H}^{t_{\delta_{1}, \beta_{1}, \alpha_{1}}}\left(\alpha_{0}\right)$ is isomorphic to $t_{\delta_{0}, \beta_{0}, \alpha_{0}}$ :

$$
\left(M, \in, A_{\delta_{1}, \beta_{1}, \alpha_{1}} \cap M, \partial_{\delta_{1}, \beta_{1}, \alpha_{1}}\right) \simeq\left(N_{\delta_{0}, \beta_{0}, \alpha_{0}}, \in, A_{\delta_{0}, \beta_{0}, \alpha_{0}}, \partial_{\delta_{0}, \beta_{0}, \alpha_{0}}\right),
$$

and 
(iii) if $\alpha_{0}<\alpha_{1}$, then there is no proper elementary submodel $M \prec\left(N_{\delta_{1}, \beta_{1}, \alpha_{1}}, \in\right.$ , $\left.A_{\delta_{1}, \beta_{1}, \alpha_{1}}, \partial_{\delta_{1}, \beta_{1}, \alpha_{1}}\right)$ with

$$
\begin{gathered}
\alpha_{0} \cup\left\{\alpha_{0}\right\} \subseteq M, \text { and } \\
M \cap \alpha_{1} \subseteq \beta_{0} .
\end{gathered}
$$

Roughly speaking, in level $\beta$ we have (isomorphism types of) initial segments $M$ of models of the form $\left(L_{\Delta^{\prime}}[A], \in, A, \Delta\right)$ (for some $\Delta \in\left[\xi, \omega_{2}\right)$ ), such that $M \cap \omega_{1} \leq \beta$, and $M$ is maximal w.r.t. this condition. We need to check that $T$ is a tree, its levels are countable, and that it has only $\omega_{2}$-many branches even in $V$.

The following claim is a standard calculation, but for the sake of completeness we include the proof.

Claim 4.12. Let $\delta \in\left[\xi, \omega_{2}\right)$ be fixed, $\beta_{0} \leq \beta_{1}<\omega_{1}$, let $\alpha_{1}=\max \left(C_{\delta} \cap\left(\beta_{1}+1\right)\right)$, $\alpha_{0}=\max \left(C_{\delta} \cap\left(\beta_{0}+1\right)\right)$. Then $\left(\beta_{0}, t_{\delta, \beta_{0}, \alpha_{0}}\right) \leq_{T}\left(\beta_{1}, t_{\delta, \beta_{1}, \alpha_{1}}\right)$.

Moreover, the embedding $\pi_{\beta_{0}, \beta_{1}}: N_{\delta, \beta_{0}, \alpha_{0}} \rightarrow N_{\delta, \beta_{1}, \alpha_{1}}$ is unique.

Proof. First observe that by (4.4) and (4.7) for $\delta \in\left[\xi, \omega_{2}\right), \alpha_{0}<\alpha_{1}$

$$
\mathfrak{H}^{\left(M_{\delta, \alpha_{1}}, \in, A, \delta\right)}\left(\alpha_{0}\right)=\mathfrak{H}^{\left(L_{\delta^{\prime}}[A], \in, A, \delta\right)}\left(\alpha_{0}\right)=M_{\delta, \alpha_{0}},
$$

therefore since $\beta_{1}<\omega_{1}$ is such that $\alpha_{1}=\max \left(C_{\delta} \cap\left(\beta_{1}+1\right)\right)$, then applying (the restriction of) the collapsing isomorphism $\pi_{\delta, \alpha_{1}}$ to the left side, we obtain

$$
\left(\mathfrak{H}^{\left(N_{\delta, \beta_{1}, \alpha_{1}}, \in, A_{\delta, \beta_{1}, \alpha_{1}}, \partial_{\delta, \beta_{1}, \alpha_{1}}\right)}\left(\alpha_{0}\right), \in\right) \simeq\left(M_{\delta, \alpha_{0}}, \in\right)
$$

and because $\beta_{0}<\beta_{1}$ is such that $\alpha_{0}=\max \left(C_{\delta} \cap\left(\beta_{0}+1\right)\right)$, then applying the isomorphism $\pi_{\delta, \alpha_{0}}$ to the right side (which fixes $\alpha_{0}$ ) we obtain

$$
\left(\mathfrak{H}^{\left(N_{\delta, \beta_{1}, \alpha_{1}}, \in, A_{\delta, \beta_{1}, \alpha_{1}}, \partial_{\delta, \beta_{1}, \alpha_{1}}\right)}\left(\alpha_{0}\right), \in\right) \simeq\left(N_{\delta, \alpha_{0}, \beta_{0}}, \in\right) .
$$

Finally, since $\pi_{\delta, \alpha_{1}}(A)=A_{\delta, \beta_{1}, \alpha_{1}}, \pi_{\delta, \alpha_{0}}(A)=A_{\delta, \beta_{0}, \alpha_{0}}$, and $\pi_{\delta, \alpha_{1}}(\delta)=\partial_{\delta, \beta_{1}, \alpha_{1}}$, $\pi_{\delta, \alpha_{0}}(\delta)=\partial_{\delta, \beta_{0}, \alpha_{0}}$, we have

$$
\left(\mathfrak{H}^{N_{\delta, \beta_{1}, \alpha_{1}}}\left(\alpha_{0}\right), \in A_{\delta, \beta_{1}, \alpha_{1}}, \partial_{\delta, \beta_{1}, \alpha_{1}}\right)
$$

is isomorphic to $\left(N_{\delta, \beta_{0}, \alpha_{0}}, \in, A_{\delta, \beta_{0}, \alpha_{0}}, \partial_{\delta, \beta_{0}, \alpha_{0}}\right)$,

therefore $(\mathrm{ii})$ holds. The uniqueness easily follows from the facts that the embedding of $\left(N_{\delta, \beta_{0}, \alpha_{0}}, \in, A_{\delta, \beta_{0}, \alpha_{0}}, \partial_{\delta, \beta_{0}, \alpha_{0}}\right)$ has to fix the ordinals less than $\alpha_{0}$, and elementary embeddings uniquely extend to Skolem-hulls.

For (iii) suppose that $\alpha_{0}<\alpha_{1}$, and note that

$$
\left(N_{\delta, \beta_{1}, \alpha_{1}}, \in\right) \models \text { ' } \alpha_{1} \text { is the least uncountable ordinal, } \alpha_{0} \text { is countable', }
$$

and for $M \prec\left(N_{\delta, \beta_{1}, \alpha_{1}}, \in, A_{\delta, \beta_{1}, \alpha_{1}}, \partial_{\delta, \beta_{1}, \alpha_{1}}\right)$ if $\alpha_{0} \cup\left\{\alpha_{0}\right\} \subseteq M$ then consider the corresponding submodel $M^{\prime} \prec\left(M_{\delta, \alpha_{1}}, \in, A, \delta\right)$, for which $M^{\prime} \supseteq M_{\delta, \alpha_{0}+1}$. But (recalling (4.8) $)$ since $\max \left(C_{\delta} \cap\left(\beta_{0}+1\right)\right)=\alpha_{0}$ we obtain $\beta_{0} \cup\left\{\beta_{0}\right\} \subseteq M^{\prime} \subseteq M_{\delta, \alpha_{1}}$, that can happen only if $\beta_{0}$ is smaller than the least uncountable ordinal in $N_{\delta, \beta_{1}, \alpha_{1}}$, $\alpha_{1}$. But then $\beta_{0} \in M \cap \alpha_{1}$.

The next claim will verify that $T$ is a tree of height $\omega_{1}$ (for the transitivity of $\leq_{T}$ use the claim two times).

Claim 4.13. For a fixed $\delta_{1} \in\left[\xi, \omega_{2}\right), \beta_{0} \leq \beta_{1}<\omega_{1}$, let $\alpha_{1}=\max \left(C_{\delta_{1}} \cap\left(\beta_{1}+1\right)\right.$, and fix arbitrary $\alpha_{0} \in \omega_{1}, \delta_{0} \in\left[\xi, \omega_{2}\right)$. Then $\left(\beta_{0}, t_{\delta_{0}, \beta_{0}, \alpha_{0}}\right) \leq_{T}\left(\beta_{1}, t_{\delta_{1}, \beta_{1}, \alpha_{1}}\right)$ iff $t_{\delta_{0}, \beta_{0}, \alpha_{0}}=t_{\delta_{1}, \beta_{0}, \max \left(C_{\delta_{1} \cap\left(\beta_{0}+1\right)}\right)}$. 
Proof. We only have to check the 'only if' part, but first observe that Definition 4.11 clearly implies that up to isomorphism there exists only one $t$ for which $\left(\beta_{0}, t\right) \leq\left(\beta_{1}, t_{\delta_{1}, \beta_{1}, \alpha_{1}}\right)$. Now the claim is the consequence of the fact that $t_{\delta_{*}, \beta_{0}, \alpha_{*}} \neq t_{\delta_{* *}, \beta_{0}, \alpha_{* *}}$ implies that they are not isomorphic as structures of the language $\mathcal{L}_{\in}\left(R_{A}, c_{\partial}\right)$ : For transitive sets $N$ and $N^{\prime}$ with $X, \partial \in N, X^{\prime}, \partial^{\prime} \in N^{\prime}$ the structures $(N, \in, X, \partial),\left(N^{\prime}, \in, X^{\prime}, \partial^{\prime}\right)$ are isomorphic if and only if $N=N^{\prime}$, $X=X^{\prime}$ and $\partial=\partial^{\prime}$ (since by the uniqueness of the Mostowski collapse we know that $(N, \in) \simeq\left(N^{\prime}, \in\right)$ iff $\left.N=N^{\prime}\right)$.

Lemma 4.14. For each $\beta<\omega_{1}$ the $\beta$ 'th level of $T$ is countable.

Proof. By Claim 4.13 we have that the $\beta$ 'th level of $T$ is

$$
\left.T_{\leq \beta} \backslash T_{<\beta}=\left\{\left(\beta, t_{\delta, \beta, \alpha}\right): \delta \in\left[\xi, \omega_{2}\right), \alpha=\max \left(C_{\delta} \cap(\beta+1)\right)\right\}\right\} .
$$

For a fixed $\delta \in\left[\xi, \omega_{2}\right)$ fix $\alpha=\max \left(C_{\delta} \cap(\beta+1)\right)$ too, and consider the structure

$$
t_{\delta, \beta, \alpha}=\left(N_{\delta, \beta, \alpha}, \in, A_{\delta, \beta, \alpha}, \partial_{\delta, \beta, \alpha}\right),
$$

where $N_{\delta, \beta, \alpha}$ is the Mostowski collapse of $\left(M_{\delta, \alpha}, \in\right)$ (by the isomorphism $\pi_{\delta, \alpha}$ ), and $A_{\delta, \beta, \alpha}=A \cap \alpha$. Now (4.6) states $M_{\delta, \alpha} \prec\left(L_{\delta^{\prime}}, \in, A\right)$ then (recalling $M_{\delta, \alpha} \cap \omega_{1}=\alpha$, and $\pi_{\delta, \alpha}\left\lceil\alpha=\mathrm{id}_{\alpha}\right.$ ) by Lemma 4.4

$$
N_{\delta, \beta, \alpha}=L_{\gamma}[A \cap \alpha]
$$

for some $\gamma=\gamma(\delta, \alpha) \in\left(\alpha, \omega_{1}\right)$. Now we determine an upper bound $\gamma_{\alpha}$ for the set $\left\{\gamma(\delta, \alpha): \delta \in\left[\xi, \omega_{2}\right) \wedge \alpha \in C_{\delta}\right\}$. If we have such a bound for each possible $\alpha \leq \beta$, then letting $\gamma_{\infty}$ denote $\sup \left\{\gamma_{\alpha}: \alpha \leq \beta\right\}$, we get

$$
\begin{gathered}
\left.\left.\left\{t_{\delta, \beta, \alpha}\right): \delta \in\left[\xi, \omega_{2}\right), \alpha=\max \left(C_{\delta} \cap(\beta+1)\right)\right\}\right\} \subseteq \\
\left\{\left(L_{\gamma}[A \cap \alpha], \in, A \cap \alpha, \partial\right): \gamma \leq \gamma_{\infty}, \alpha \leq \beta, \partial<\gamma\right\},
\end{gathered}
$$

which latter set is obviously countable, this will finish the proof of the lemma.

So fix $\alpha \leq \beta$ and $\delta \in\left[\xi, \omega_{2}\right)$ such that $\alpha \in C_{\delta}$. Now we have two cases depending on whether there is any (cardinal) $)^{L[A \cap \alpha]}$ in $\left(\alpha, \omega_{1}\right)$. If $\lambda \in\left(\alpha, \omega_{1}\right)$ is a cardinal in the inner model $L[A \cap \alpha]$, then for each $\delta$ if $\alpha=\max \left(C_{\delta} \cap(\beta+1)\right)$, then the transitive set $N_{\delta, \beta, \alpha}$ cannot contain $\lambda$, as $M_{\delta, \alpha}$ sees $\omega_{1}$ as the largest cardinal, and $\pi_{\delta, \alpha}\left(\omega_{1}\right)=\alpha$. This case choosing $\gamma_{\alpha}=\lambda$ works.

On the other hand, if $\left(|\alpha|^{+}\right)^{L[A \cap \alpha]}=\omega_{1}$, then we first prove that $\alpha \in C_{\delta}$ implies $(|\alpha|=\omega)^{L[A \cap \alpha]}$ : otherwise in $M_{\delta, \alpha}$, and in $N_{\delta, \beta, \alpha}$ each ordinal less than $\alpha$ are countable, thus as well in $L[A \cap \alpha]$. Then it is easy to see that the condition

$$
\text { ( } \left.\lambda \text { is the unique cardinal in }\left(\omega, \omega_{1}^{V}\right)\right)^{L[A \cap \lambda]}
$$

cannot hold for two different $\lambda$ 's, therefore $\alpha$ can be defined in $L[A]$. But then using Claim 4.5 with $X=A \cap \alpha$ we have that for each $\zeta \in\left(\alpha, \omega_{1}\right)$ there is a bijection $f_{\zeta} \in L_{\omega_{1}}[A \cap \alpha]$ between $\alpha$ and $\zeta$, therefore $\alpha$ can be defined also in $L_{\delta^{\prime}}[A]$, and $M \prec\left(L_{\delta^{\prime}}[A], \in\right)$ implies $\alpha \in M$, contradicting that $M_{\delta, \alpha} \cap \omega_{1}=\alpha$ (which holds by $\left.\alpha \in C_{\delta}\right)$. Then $(|\alpha|=\omega)^{L[A \cap \alpha]}$ and Claim 4.5 implies that there is an ordinal $\lambda<\omega_{1}$ such that there exists a bijection between $\alpha$ and $\omega$ in $L_{\lambda}[A \cap \alpha]$, implying

$$
N_{\delta, \beta, \alpha}=L_{\gamma(\delta, \alpha)}[A \cap \alpha] \subsetneq L_{\lambda}[A \cap \alpha],
$$

since $\alpha$ is uncountable in $N_{\delta, \beta, \alpha}$. This case

$$
\left\{\gamma(\delta, \alpha): \delta \in\left[\xi, \omega_{2}\right) \wedge \alpha \in C_{\delta}\right\} \subseteq \gamma_{\alpha}=\lambda,
$$

which completes the proof of Lemma 4.14. 
Now $T$ is obviously a Kurepa tree by the following fact and lemma.

Fact 4.15. The sequence $\left\langle B_{\delta}: \delta \in\left[\xi, \omega_{2}\right)\right\rangle$ lists pairwise distinct cofinal branches in $T$, where

$$
B_{\delta}=\left\{\left(\beta, t_{\delta, \beta, \max \left(C_{\delta} \cap(\beta+1)\right)}\right): \beta<\omega_{1}\right\} .
$$

Proof. We only need to prove that $B_{\delta} \neq B_{\gamma}$ if $\delta \neq \gamma$. But according to the second statement of Claim 4.12 for each $\beta<\beta^{\prime}<\omega_{1}$ there is a unique elementary embedding of $t_{\delta, \beta^{\prime}, \max \left(C_{\delta} \cap\left(\beta^{\prime}+1\right)\right)}$ to $t_{\delta, \beta, \max \left(C_{\delta} \cap(\beta+1)\right)}$, therefore there is a unique direct-limit of this elementary chain, isomorphic to $\bigcup_{\alpha \in C_{\delta}} M_{\delta, \alpha}$, which is $\left(L_{\delta^{\prime}}[A], \in\right.$ , $A, \delta$ ) by Claim 4.10 .

It is only left to prove that each branch of $T$ is of the form $B_{\delta}$ for some $\delta \in\left[\xi, \omega_{2}\right)$ (even in $V$ ). The following lemma will complete the proof of Theorem 4.1 .

Lemma 4.16. Let $B \subseteq T$ a cofinal branch in $T, B \in V$. Then $B=B_{\delta}$. for a unique $\delta_{\bullet} \in\left[\xi, \omega_{2}\right)$.

Proof. Let $t_{\delta_{\beta}, \beta, \alpha_{\beta}}=\left(N_{\delta_{\beta}, \beta, \alpha_{\beta}}, \in, A_{\delta_{\beta}, \beta, \alpha_{\beta}}, \partial_{\delta_{\beta}, \beta, \alpha_{\beta}}\right)$ denote the element in $B \cap$ $\left(T_{\leq \beta} \backslash T_{<\beta}\right)$. Working in $V$ first we define the following bonding maps: for $\gamma \leq \beta<$ $\omega_{1}$ let

$$
\pi_{\gamma, \beta}: N_{\delta_{\gamma}, \gamma, \alpha_{\gamma}} \rightarrow N_{\delta_{\beta}, \beta, \alpha_{\beta}}
$$

be the unique elementary embedding (combining Claim 4.13) and the second statement of Claim 4.12). Since elementary submodels of an elementary submodel are elementary submodels, $\pi_{\beta^{\prime}, \beta} \circ \pi_{\beta^{\prime \prime}, \beta^{\prime}}$ is an elementary embedding for each $\beta^{\prime \prime} \leq \beta^{\prime} \leq \beta<\omega_{1}$, therefore by the uniqueness

$$
\left(\forall \beta^{\prime \prime} \leq \beta^{\prime} \leq \beta<\omega_{1}\right): \pi_{\beta^{\prime}, \beta} \circ \pi_{\beta^{\prime \prime}, \beta^{\prime}}=\pi_{\beta^{\prime \prime}, \beta} .
$$

This elementary chain allows us to define the limit $D=\left(N_{\omega_{1}}, \mathbf{E}, A_{\omega_{1}}, \partial_{\omega_{1}}\right)$ of the directed system $\left\{t_{\delta_{\beta}, \beta, \alpha_{\beta}}, \pi_{\beta^{\prime}, \beta}: \quad \beta^{\prime} \leq \beta<\omega_{1}\right\}$.

Let $\pi_{\beta}: N_{\delta_{\beta}, \beta, \alpha_{\beta}} \rightarrow N_{\omega_{1}}$ be the embedding, $N_{\beta}=\operatorname{ran}\left(\pi_{\beta}\right)$ (hence $N_{\omega_{1}}=$ $\left.\bigcup_{\beta<\omega_{1}} N_{\beta}\right)$.

First note that $\left(N_{\omega_{1}}, \mathbf{E}\right)$ is well-founded, otherwise there would be an infinite E-decreasing chain in the embedded image of $N_{\delta_{\beta}, \beta, \alpha_{\beta}}$ for some (in fact, every large enough) $\beta$, contradicting that $\left(N_{\delta_{\beta}, \beta, \alpha_{\beta}}, \in\right)$ is well-founded. Now (by the E-extensionality in $N_{\omega_{1}}$ ) we can assume that $N_{\omega_{1}}$ is a Mostowski collapse, i.e. $\left(N_{\omega_{1}}, \mathbf{E}\right)=\left(N_{\omega_{1}}, \in\right)$. Then it is easy to see that if $\beta<\omega_{1}$ for the elementary embedding $\pi_{\beta}: N_{\delta_{\beta}, \beta, \alpha_{\beta}} \rightarrow N_{\omega_{1}}$ we have $\pi_{\beta}\left\lceil\alpha_{\beta}=\operatorname{id}_{\alpha_{\beta}}\right.$, and $\pi_{\beta}\left(\alpha_{\beta}\right)=\omega_{1}$, thus (recalling that $\left.A_{\delta_{\beta}, \beta, \alpha_{\beta}}=A \cap \alpha_{\beta}\right)$ we obtain $\left(N_{\omega_{1}}, \mathbf{E}, A_{\omega_{1}}, \partial_{\omega_{1}}\right)=\left(N_{\omega_{1}}, \in, A, \delta_{\bullet}\right)$ for some $\delta_{\bullet} \in\left(\omega_{1}, \omega_{2}\right)$. Now we can use Lemma 4 .4 (since $\left(N_{\delta_{\beta}, \beta, \alpha_{\beta}}, \in, A_{\delta_{\beta}, \beta, \alpha_{\beta}}\right) \models \sigma$ ), there exists $\zeta>\delta_{\bullet}$ such that

$$
N_{\omega_{1}}=L_{\zeta}[A],
$$

and then

$$
\left(N_{\omega_{1}}, \in, A, \delta_{\bullet}\right)=\left(L_{\zeta}[A], \in, A, \delta_{\bullet}\right) .
$$

Now because the formula $\sigma^{\prime} \in \mathcal{L}_{\in}\left(R_{A}, c_{\partial}\right)$ from Claim 4.9 holds in $\left(L_{\delta^{\prime}}[A], \in, A, \delta\right)$ (for each $\delta \in\left[\xi, \omega_{2}\right)$ ) (for our mapping $\delta \mapsto \delta^{\prime}$ from Definition 4.8) and therefore also in $M_{\delta, \alpha}$ 's, $N_{\delta, \beta, \alpha}$ 's $\left(\delta \in\left[\xi, \omega_{2}\right)\right)$, so it must hold in $\left(N_{\omega_{1}}, \in, A, \delta_{\bullet}\right)$, which means that $\delta_{\bullet} \geq \xi$, and $\zeta=\delta_{\bullet}^{\prime}$, i.e.

$$
\left(N_{\omega_{1}}, \in, A, \delta_{\bullet}\right)=\left(L_{\delta_{\bullet}^{\prime}}[A], \in, A, \delta_{\bullet}\right),
$$


Finally, we have to prove that for each $\beta<\omega_{1}$

$$
t_{\delta_{\beta}, \beta, \alpha_{\beta}}=\left(N_{\delta_{\beta}, \beta, \alpha_{\beta}}, \in, A_{\delta_{\beta}, \beta, \alpha_{\beta}}, \partial_{\delta_{\beta}, \beta, \alpha_{\beta}}\right)=t_{\delta_{\bullet}, \beta, \max \left(C_{\delta_{\bullet}} \cap(\beta+1)\right)}
$$

by arguing (having $\beta$ fixed) that for a large enough $\gamma$

$$
\left(\beta, t_{\delta_{\bullet}, \beta, \max \left(C_{\delta_{\bullet}} \cap(\beta+1)\right)}\right) \leq_{T}\left(\gamma, t_{\delta_{\gamma}, \gamma, \alpha_{\gamma}}\right) .
$$

Let $\alpha=\max \left(C_{\delta_{\bullet}} \cap(\beta+1)\right), \alpha^{\prime}=\min \left(C_{\delta_{\bullet}} \backslash(\beta+1)\right), \beta^{\prime}=\alpha^{\prime}$, and consider the models $M_{\delta_{\bullet}, \alpha}, M_{\delta_{\bullet}, \alpha^{\prime}} \prec\left(L_{\delta_{\bullet}^{\prime}}[A], \in, A, \delta_{\bullet}\right)$. Choose $\gamma \geq \beta^{\prime}, \gamma<\omega_{1}$ so that $N_{\gamma}=\pi_{\gamma}\left[N_{\delta_{\gamma}, \gamma, \alpha_{\gamma}}\right] \supseteq M_{\delta_{\bullet}, \alpha^{\prime}}$. Then

$$
\alpha_{\gamma} \geq \alpha^{\prime}>\beta+1, \text { and }
$$

$\alpha^{\prime} \cup\left\{\omega_{1}\right\} \subseteq N_{\gamma} \prec\left(L_{\delta_{\bullet}^{\prime}}[A], \in, A, \delta_{\bullet}\right)$ with (4.7) imply

$$
\mathfrak{H}^{\left(N_{\gamma}, \in, A \cap N_{\gamma}, \delta_{\bullet}\right)}(\alpha)=\mathfrak{H}^{\left(L_{\delta_{\bullet}^{\prime}}[A], \in, A, \delta_{\bullet}\right)}(\alpha)=M_{\delta_{\bullet}, \alpha} .
$$

Therefore in $\left(N_{\gamma}, \in, A \cap N_{\gamma}, \delta_{\bullet}\right) \simeq\left(N_{\delta_{\gamma}, \gamma, \alpha_{\gamma}}, \in, A_{\delta_{\gamma}, \gamma, \alpha_{\gamma}}, \partial_{\delta_{\gamma}, \gamma, \alpha_{\gamma}}\right)$ there is an elementary submodel isomorphic to $\left(M_{\delta_{\bullet}, \alpha}, \in, A \cap M_{\delta_{\bullet}, \alpha}, \delta_{\bullet}\right)$, which latter is isomorphic to $\left(N_{\delta_{\bullet}, \beta, \alpha}, \in, A \cap \alpha, \partial_{\delta_{\bullet}, \beta, \alpha}\right)$, thus $[(i i)$ from Definition 4.11 holds.

Similarly, using also (4.10) and the definitions of $\alpha, \alpha^{\prime}$

$$
\mathfrak{H}^{\left(N_{\gamma}, \in, A \cap N_{\gamma}, \delta_{\bullet}\right)}(\alpha+1)=M_{\delta_{\bullet}, \alpha+1}=M_{\delta_{\bullet}, \alpha^{\prime}} \supseteq \alpha^{\prime} \supseteq \beta \cup\{\beta\},
$$

ans since the isomorphism between $\left(N_{\gamma}, \in, A \cap N_{\gamma}, \delta_{\bullet}\right)$ and $\left(N_{\delta_{\gamma}, \gamma, \alpha_{\gamma}}, \in\right.$ , $\left.A_{\delta_{\gamma}, \gamma, \alpha_{\gamma}}, \partial_{\delta_{\gamma}, \gamma, \alpha_{\gamma}}\right)$ fixes the ordinals less than or equal to $\alpha^{\prime}$ we obtain

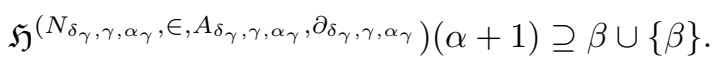

Therefore recalling (4.15) we obtain that (iii) (of Definition 4.11) holds as well.

\section{REFERENCES}

[HM19] Yair Hayut and Sandra Muller, Perfect subtree property for weakly compact cardinals, preprint (2019), arxiv:math/1910.05159.

[JS92] Renling Jin and Saharon Shelah, Planting Kurepa trees and killing Jech-Kunen trees in a model by using one inaccessible cardinal, Fundamenta Mathematicae 141 (1992), 287-296, arxiv:math/9211214.

[JS93] - A model in which there are Jech-Kunen trees but there are no Kurepa trees, Israel Journal of Mathematics 84 (1993), 1-16, arxiv:math/9308218.

[JS94] Essential Kurepa trees versus essential Jech-Kunen trees, Annals of Pure and Applied Logic 69 (1994), 107-131, arxiv:math/9401217.

[Kan03] A. Kanamori, The higher infinite: Large cardinals in set theory from their beginnings, Springer monographs in mathematics, Springer, 2003.

[Kos05] Piotr Koszmider, Kurepa trees and topological non-reflection, Topology and its Applications 151 (2005), 77-98.

[Kun78] Kenneth Kunen, Saturated ideals, Journal of Symbolic Logic 43 (1978), 65-76.

[Kun83] _ Set Theory: An introduction to independence proofs, Studies in Logic and the Foundations of Mathematics, vol. 102, North-Holland Publishing Co, 1983.

[Kun13] _ Set Theory, Studies in logic. Mathematical logic and foundations, vol. 34, College Publications, 2013.

[Poo] Mark Poor, On the spectra of cardinalities of branches of Kurepa trees, Archive for Mathematical Logic accepted, arxiv:math/1706.01409.

[Sil67] Jack Silver, The independence of Kurepa's conjecture and two-cardinal conjectures in model theory, Axiomatic Set Theory, Proc. Symp in Pure Math., vol. XIII, 1967, pp. 383390.

[SS17] Dima Sinapova and Ioannis Souldatos, Kurepa trees and spectra of $\mathcal{L}_{\omega_{1}, \omega}$-sentences, preprint (2017), arxiv:math/1705.05821. 
Eötvös Loránd University, Institute of Mathematics, PÁzmány Péter s. 1/C, 1117 Budapest, Hungary

Email address: sokmark@caesar.elte.hu

Einstein Institute of Mathematics, Edmond J. Safra Campus, Givat Ram, The HeBrew University of Jerusalem, Jerusalem, 91904, Israel, and, Department of Mathematics, Hill Center - Busch Campus, Rutgers, The State University of New Jersey, 110 Frelinghuysen Road, Piscataway, NJ 08854-8019 USA

Email address: shelah@math.huji.ac.il

URL: http://shelah.logic.at 